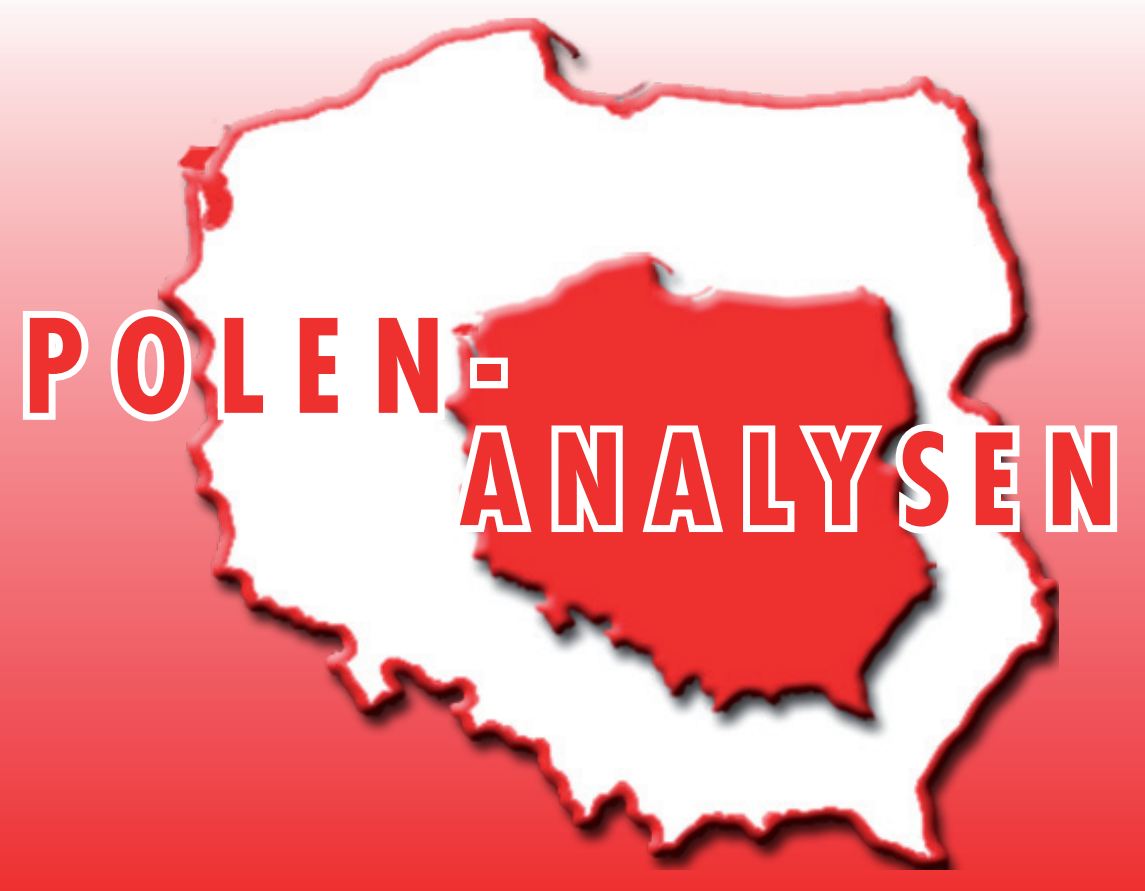

www.laender-analysen.de/polen

\title{
REGIERUNGSWECHSEL IN POLEN
}

ANALYSE

Die neue Regierung von Ewa Kopacz auf dem Prüfstand

Stefan Garsztecki, Chemnitz

DOKUMENTATION

Exposé der Ministerpräsidentin Ewa Kopacz vom 1. Oktober 2014

(Auszüge aus dem Stenogramm)

- UMFRAGE

Einstellungen zur Regierung von Ewa Kopacz und zu Vorgängerregierungen

CHRONIK

21. Oktober - 3. November 2014 


\title{
Die neue Regierung von Ewa Kopacz auf dem Prüfstand
}

\author{
Stefan Garsztecki, Chemnitz
}

\section{Zusammenfassung}

Gut ein Jahr vor den nächsten Parlamentswahlen in Polen hat das Land eine neue Regierung. Nach der Wahl von Donald Tusk zum neuen Präsidenten des Europäischen Rates ab dem 1. Dezember 2014 wurde mit Ewa Kopacz die stellvertretende Parteivorsitzende der Bürgerplattform (Platforma Obywatelska - PO) und Sejmmarschallin neue Regierungschefin. Für Tusk war es ein Abgang in schwierigen politischen Zeiten, der Ewa Kopacz den Start nicht gerade erleichtert. Ihr Exposé und erste Personalentscheidungen zeigen, wie sie die ihr zur Verfügung stehende Zeit bis zu den nächsten Parlamentswahlen im Herbst 2015 nutzen möchte. Mit Pragmatismus, Bürgernähe und einer starken Regierungsmannschaft, die auch Schwergewichte der $P O$ umfasst, möchte sie die Bürger von ihrem Kurs überzeugen. Ersten Umfragen zufolge ist ihr zumindest ein guter Start gelungen.

$\mathrm{D}_{\mathrm{s}}^{\mathrm{ic}}$ Wahl des polnischen Ministerpräsidenten Donald Tusk als Nachfolger des Belgiers Herman van Rompuy zum Präsidenten des Europäischen Rates zum 1. Dezember 2014 hat eine hohe Symbolkraft sowohl für die Europäische Union als auch für Polen selbst. Fast auf den Tag genau 25 Jahre nach dem Fall des Eisernen Vorhangs und gut zehn Jahre nach der ersten großen Osterweiterung der EU bekleidet mit Tusk nach dem polnischen Präsidenten des EU-Parlamentes Jerzy Buzek (von Juli 2009 bis Januar 2012) erneut ein Osteuropäer ein Spitzenamt in Europa - und dieses Mal im Zentrum der Macht. Für die EU ist der sehr erfahrene Tusk, der ab Herbst 2007 Regierungschef in Polen war, mit Blick auf den nach wie vor nicht gelösten Konflikt in der Ukraine und das schwierige Verhältnis zu Russland eine sehr gute Wahl, zumal auch das Verhältnis zu Bundeskanzlerin Angela Merkel als ausgezeichnet gilt. Ob sich der manchmal etwas steife Tusk im Brüsseler Machtpoker zurechtfinden wird, bleibt gleichwohl abzuwarten. In der politischen Landschaft Polens war Tusk trotz oder vielleicht auch gerade wegen seines im Ton konzilianten Auftretens über Jahre sowohl in seiner Partei, der Bürgerplattform, als auch im Land fast ohne nennenswerte Konkurrenz, da die oft scharfen Auftritte von Oppositionsführer Jarosław Kaczyński von der Partei Recht und Gerechtigkeit (Prawo i Sprawiedliwość $P i S$ ) über die mangelnde Entscheidungsfreudigkeit des Ministerpräsidenten hinwegtäuschten und ihn alternativlos erscheinen ließen.

Zuletzt hatte Donald Tusk dennoch Zeichen von Amtsmüdigkeit gezeigt. Bei den Wahlen zum Europäischen Parlament Ende Mai 2014 hatte sich die PO zwar noch knapp vor PiS behaupten können, allerdings hatte die Partei mehr als 12 Prozent verloren und liegt nun mit 19 Abgeordneten im Europäischen Parlament gleichauf mit PiS. Zudem hatte die Regierung seit Beginn des Jahres 2012 mit zunehmender Kritik in der öffent- lichen Wahrnehmung zu kämpfen und hatte in Umfragen bis zum Ende der Amtszeit von Donald Tusk mehr Gegner als Anhänger. Dazu trugen verschiedene politische Affären bei, von denen die letzte im Juni 2014 die Regierung an den Rand des Abgrundes brachte. Das konservative Wochenmagazin »Wprost « hatte in diesem Monat von Kellnern heimlich aufgenommene Gespräche zwischen prominenten Politikern in einem Warschauer Restaurant veröffentlicht. Von diesen Treffen zwischen Innenminister Bartłomiej Sienkiewicz und Notenbankpräsident Marek Belka bzw. zwischen Außenminister Radosław Sikorski und dem ehemaligen Finanzminister Jacek Rostowski im Jahr 2013 wurden politische Absprachen und Aussagen publik, die einen Skandal verursachten. Demnach machte der Notenbankpräsident seine Unterstützung der Regierung von der Entlassung des damaligen Finanzministers Rostowski abhängig. Außenminister Sikorski hingegen bezeichnete das Bündnis mit den USA als wertlos. Neben dem Inhalt der Gespräche war auch die Sprache der Spitzenpolitiker selbst kritisiert worden. Zwar konnte Tusk eine Vertrauensfrage Ende Juni 2014 im Sejm gewinnen, aber die Lust auf das Regieren dürfte es ihm weiter genommen haben, zumal das öffentliche Vertrauen in die Regierung kurzfristig weiter abnahm. Nimmt man zu den schlechten Umfragedaten und der "Abhöraffäre« noch die innerparteilichen Querelen hinzu, dann dürfte Tusk die Chance, Ratspräsident zu werden, fast schon als Erlösung betrachtet haben. Noch vor der letzten Parlamentswahl im Herbst 2011 in Polen war im Oktober 2010 der parteiinterne Kritiker Janusz Palikot aus der $P O$ ausgetreten, um bei den nächsten Parlamentswahlen mit einer eigenen Gruppierung, der Palikot-Bewegung (Ruch Palikota), rund 10 Prozent der Stimmen zu erlangen. Mittlerweile liegt diese Gruppierung in Umfragen aber deutlich unter der 5-Prozent-Hürde. Gravierender waren wohl die Attacken von Jarosław Gowin, der 
dem konservativen Flügel innerhalb der $P O$ zugerechnet wurde. Von 2011 bis zu seiner Abberufung durch Tusk übte er das Amt des Justizministers aus, lag aber in normativen Fragen mit Tusk und großen Teilen der Partei über Kreuz. So lehnte er sowohl eingetragene Lebenspartnerschaften, die von Tusk unterstützt wurden, wie auch das Einfrieren von Ei- und Samenzellen und die Invitro-Befruchtung für unverheiratete Paare ab, während dies die Parteilinie war und Polen als einziges EU-Mitgliedsland noch keine gesetzliche Regelung dieser Frage aufweist. Donald Tusk war es laut eigener Aussage leid, dies immer wieder öffentlich kommentieren zu müssen, und berief daher Gowin als Justizminister ab. Nachdem dieser im August 2013 die Wahl zum Vorsitzenden der $P O$ gegen Tusk verloren hatte, trat er aus der Partei aus und gründete seine eigene Partei. Polen Gemeinsam (Polska Razem) steht normativ PiS näher und arbeitet mit dieser Partei seit dem Sommer 2014 auch zusammen.

Es waren also nicht nur die europäischen Versuchungen, die Tusk den Wechsel nach Brüssel schmackhaft machten, sondern auch die innerparteilichen Querelen und die wenig komfortable Situation der Regierung ein Jahr vor den Sejmwahlen im Herbst 2015. Damit stellt sich auch die Frage, welche Chance die neue Regierung von Ministerpräsidentin Ewa Kopacz ein Jahr vor den nächsten Wahlen hat und mit welchen eigenen Akzenten sie versucht, politisches Profil zu gewinnen. Als Generationenwechsel, der im Übrigen besser in der Mitte einer Amtszeit durchzuführen wäre, kann die Stabsübergabe an Ewa Kopacz zudem nicht betrachtet werden, ist doch die neue Ministerpräsidentin ein knappes halbes Jahr älter als Tusk und wird Anfang Dezember 58 Jahre alt. Mit Blick auf die anstehenden Wahlen - die Kommunalund Regionalwahlen im November dieses Jahres, Präsidentschafts- und Parlamentswahlen im Frühjahr bzw. Herbst des kommenden Jahres - wird von ihr nicht nur erfolgreiches Regieren, sondern auch eine Erneuerung der Bürgerplattform erwartet.

\section{Ministerpräsidentin Ewa Kopacz und das Parteikalkül}

Ewa Kopacz stand bisher eher im Schatten des langjährigen Ministerpräsidenten Tusk, obgleich sie bereits höchste Staatsämter innehatte. Die ausgebildete Kinderärztin war im ersten Kabinett Tusk Gesundheitsministerin und bekleidete bis zu ihrer Wahl zur Ministerpräsidentin das Amt des Sejmmarschalls. In den 1990er Jahren gehörte sie der aus dem Intellektuellen-Milieu der Gewerkschaft Solidarność hervorgegangenen liberalen Freiheitsunion (Unia Wolności-UW) an, bevor sie 2001 zur $P O$ wechselte, für die sie ab 2001 Abgeordnete im Sejm war. Seit Herbst 2010 ist sie zudem Vizevorsitzende der Bürgerplattform und enge Vertraute von Donald
Tusk, so dass ihre zügige Nominierung folgerichtig war. Am 22. September 2014 wurde sie von Staatspräsident Bronisław Komorowski zur Ministerpräsidentin berufen und zugleich ihr Kabinett vereidigt. Sie steht damit der Koalitionsregierung aus PO und Polnischer Bauernpartei (Polskie Stronnictwo Ludowe - PSL) vor.

Mit ihrer Regierungsmannschaft präsentierte Ministerpräsidentin Kopacz auch gleich zwei erste Überraschungen. Zum einen berief sie die einer breiteren Öffentlichkeit nahezu unbekannte, ihr aber vertraute PO-Abgeordnete Teresa Piotrowska zur neuen Innenministerin als Nachfolgerin des parteilosen Bartłomiej Sienkiewicz, der in der "Abhöraffäre« vom Juni dieses Jahres nicht die beste Figur abgegeben hatte, und zum anderen den ehemaligen Rivalen von Donald Tusk und in der $P O$ nach wie vor sehr populären Grzegorz Schetyna zum Außenminister. Er folgt damit Radosław Sikorski, der Ewa Kopacz im Amt des Sejmmarschalls beerbt. Da Elżbieta Bieńkowska, Ministerin für Infrastruktur und stellvertretende Ministerpräsidentin, im September 2014 von EU-Kommissionspräsident JeanClaude Juncker zur EU-Kommissarin berufen wurde, wurde Verteidigungsminister Tomasz Siemoniak neuer stellvertretender Regierungschef. Ein Schwergewicht ist auch der stellvertretende Parteichef Cezary Grabarczyk, der dank sehr guter Wahlergebnisse in den Parlamentswahlen eine starke Position in der Partei hat und der als Justizminister u. a. eine Regelung zur In-vitro-Fertilisation durch den Sejm bringen muss. Chef der Kanzlei der Ministerpräsidentin bleibt wie bei ihrem Vorgänger der parteilose Jacek Cichocki, während für den langjährigen Finanzminister Jacek Rostowski kein Platz in der Regierung mehr war. Seine Ernennung zum Staatssekretär in der Staatskanzlei blockierte die PSL, die seine Rückkehr im Rang eines Ministers auch mit Blick auf den Koalitionsproporz und seine Entscheidungen als Finanzminister nicht mehr wünschte. Alles in allem gehören der neuen Regierung fünf neue Minister von insgesamt 18 Regierungsmitgliedern an, so dass sich das Revirement im Rahmen hielt.

Ewa Kopacz betonte in ersten Äußerungen zur Regierungsmannschaft, dass ihr das Team wichtig sei. Zudem möchte sie das verlorene Vertrauen in die Regierung und in die Politik zurückgewinnen, was wohl die Wahl des populären Grabarczyk und Schetynas zu Ministern mit erklärt. Zugleich versucht sie im Vorfeld der Wahlen die Unruhe in der $P O$ zu mindern, indem sie mit ihnen zwei wichtige Akteure in die Regierungsdisziplin einbindet.

\section{Das Exposé: Pragmatismus und Bürgernähe} In ihrem im Sejm am 1. Oktober vorgestellten Exposé erklärte die neue Ministerpräsidentin es zu ihrer wichtigsten Aufgabe, das Vertrauen in die politischen Institu- 
tionen, und hier vor allem in den Sejm, zu stärken. Seit Jahren wird die Arbeit der beiden Parlamentskammern Sejm und Senat - von den Polen wenig geschätzt, und auch das Vertrauen in Politiker und die Zufriedenheit mit dem Funktionieren der Demokratie sind seit Jahren auf sehr niedrigem Niveau. Ewa Kopacz betont daher in diesem Zusammenhang, dass sie für die schweigende Mehrheit der Polen arbeiten möchte, die vom Parteiengezänk genug hätte.

Nach diesen allgemeinen Ausführungen beschränkte sich das knappe, 45 Minuten dauernde Exposé auf Schwerpunkte in den einzelnen Politikbereichen. Ewa Kopacz begann mit Polens Position in der EU, verwies auf die enormen Summen, die Polen aus den EU-Töpfen im Rahmen der Kohäsionspolitik erhält, und bekannte sich zu einer aktiven Rolle Polens in der EU. Ferner warb sie für eine Freihandelszone zwischen der EU und den USA und befürwortete auch klar eine zügige Einführung des Euro, sobald Polen die Bedingungen erfüllen wird, da der Euro positiv für die polnische Wirtschaft sei - ein Akzent gegen PiS, die sich gegen eine schnelle Einführung des Euro ausspricht und für ein Referendum optiert. Als zentral hob Ewa Korpacz auch Fragen der äußeren Sicherheit hervor. Sie möchte die Ausgaben im Wehretat schon im Jahr 2015 erhöhen und die Armee modernisieren, was möglicherweise einer der Gründe ist, dass Verteidigungsminister Siemoniak einer von zwei Vizeregierungschefs wurde - neben Wirtschaftsminister Janusz Piechociński, dem das Amt als Chef des Koalitionspartners PSL zugefallen ist. Für Firmen, die vom russischen Embargo betroffen sind, plant die Regierung ein Antikrisengesetz, der Bergbau soll eine rentable Branche werden und schließlich soll auch der Ausbau der Infrastruktur mit dem Bau von Autobahnen und Schnellstraßen weiter vorangetrieben werden. Ein weiterer Schwerpunkt des Exposés war die Sozialpolitik. Hier möchte Ewa Kopacz den Ausbau von Krippen und Kindergärten unterstützen und ab 2016 den Elternurlaub für alle Eltern, beschäftigt oder nicht, einführen. Ferner soll die noch von der Vorgängerregierung Tusk beschlossene Rentenanpassung im Jahr 2015 erfolgen. Für die Hochschulen sind höhere Ausgaben vorgesehen: Der Anteil für die Hochschulen soll bis 20202 Prozent des Bruttoinlandsprodukts betragen. Zugleich soll ein Praktikumsprogramm in öffentlichen Einrichtungen für Studenten gestartet und ein Studium im Ausland für die begabtesten Studierenden gefördert werden, unter der Bedingung, dass sie anschließend fünf Jahre in Polen arbeiten. Zum Schluss ihrer Ausführungen bat die neue Ministerpräsidentin nicht um einhundert ruhige Tage, sondern um Zusammenarbeit in für Polen wichtigen Fragen wie nationale Verteidigung, Energiesicherheit und die Zukunft der polnischen Kohle.
Ein interessanter Aspekt gleich im ersten Teil der Regierungserklärung war nach einer Würdigung ihres Vorgängers Donald Tusk der Appell an den Oppositionsführer Jarosław Kaczyński, »den Fluch des Hasses (gegen Tusk) von Polen zu nehmen«.

Die Reaktionen auf das Exposé waren gemischt. Naturgemäß kritisierte die Opposition die Regierungschefin. Leszek Miller, Vorsitzender der Demokratischen Linksallianz (Sojusz Lewicy Demokratycznej-SLD), warf ihr vor, die Versäumnisse der letzten sieben Jahre der $P O$ geführten Regierungen innerhalb eines Jahres nachholen zu wollen, und auch $P i S$ sprach ihr das moralische Recht $\mathrm{ab}$, an der Spitze der Regierung zu stehen, zumal sie russischen Aussagen zur Untersuchung der Flugzeugkatastrophe von Smolensk Glauben geschenkt habe, als sie Gesundheitsministerin gewesen war. Eine Rolle spielt in konservativen Kreisen und Blogs aber auch der Umstand, dass Ewa Kopacz in den 1980er Jahren der Vereinigten Bauernpartei (Zjednoczone Stronnictwo Ludowe - ZSL), einer Blockpartei, angehört hatte, wie der Sprecher des Koalitionspartners PSL, Krzysztof Kosiński, publik machte. Das trifft allerdings auch auf andere Spitzenpolitiker wie zum Beispiel den Vizeregierungschef Piechociński selbst zu und galt bisher nicht als ehrenrührig.

Die Kommentatoren in den Zeitungen gingen etwas differenzierter mit dem Exposé um. Jacek Żakowski von der einflussreichen Wochenzeitung »Polityka« lobte Ewa Kopacz nachdrücklich für den Appell an Kaczyński, die Aussagen zur Energiepolitik und den Realismus des Exposés. Sergiusz Trzeciak kritisierte in derselben Zeitung aber die fehlende Strategie der Ministerpräsidenten und den häufigen Verweis auf die Umsetzung der Pläne ab dem Jahr 2016, d. h. nach einem möglichen Wahlsieg im kommenden Jahr. Allerdings ist der Vorwurf, dass Ewa Kopacz sich in Details verloren habe, kaum haltbar, wenn man sie mit ihrem Vorgänger vergleicht, dauerte sein Exposé nach der Übernahme der Regierung im Jahr 2007 doch über drei Stunden!

Bereits im Oktober hat Ewa Kopacz mit Besuchen in den Ministerien begonnen, um sich einen Überblick über die Arbeit der Ressorts und der Vizeminister zu verschaffen. Dies ist sicherlich ein Versuch, als energische Regierungschefin wahrgenommen zu werden, nicht zuletzt um das verlorene Vertrauen in die Regierung im Besonderen und in die Politik im Allgemeinen wiederzugewinnen. Zudem waren mit dem Abgang von Tusk auch die Vizeminister zurückgetreten, so dass hier letztlich auch Personalentscheidungen über politische Spitzenbeamte fallen werden, wie sie nach Regierungswechseln üblich sind. Ungewöhnlich ist eher, dass die Regierungschefin diese Überprüfungen selber mittels Besuchen in den Ressorts durchführt und sich für diese Prozedur drei Wochen Zeit nimmt. 
Auch wenn die Regierung erst sechs Wochen im Amt ist, haben einige Entscheidungen bzw. Vorfälle doch bereits Schlagzeilen gemacht. Hier ist in erster Linie die Haltung der polnischen Regierung auf dem Klimagipfel der EU in Brüssel am 23. und 24. Oktober 2014 anzuführen. Wie in ihrem Exposé angeführt, möchte Ewa Kopacz den polnischen Bergbau konkurrenzfähig erhalten und eine Verteuerung der Energie vermeiden. Entsprechend setzte sie sich auf dem Gipfel dafür ein, dass Energie in Polen nicht teurer wird. Zwar beschloss der EU-Rat auf seinem Treffen eine Reduzierung der Treibhausgase im Vergleich zu 1990 um 40 Prozent bis 2030 und soll auch der Anteil der erneuerbaren Energie ebenso wie die Energieeffizienz um 27 Prozent zunehmen, aber für ärmere Länder wie Polen mit einem Bruttosozialprodukt unter 60 Prozent des EU-Durchschnitts sind Ausnahmen im Rahmen einer sogenannten Flexibilitätsklausel vorgesehen. So kann Polen bis zum Jahr 2030 Emissionsgenehmigungen kostenlos auf seine Energieunternehmen übertragen und auf diese Weise die Energiepreise nach Ansicht der Regierung stabil halten. Unabhängig davon, dass die EU-Klimaziele nach wie vor nicht ehrgeizig genug sein dürften, ist für Polen die Situation ausgesprochen schwierig, da rund 90 Prozent seines Energiebedarfs mit Kohle gedeckt werden. Die Regierung bewertete daher das Ergebnis von Brüssel als Erfolg der Regierung. Für $P i S$ ist die erreichte Vereinbarung aber nicht ausreichend und Oppositionsführer Kaczyński hat für den Fall eines Regierungswechsel eigene Bemühungen um ein opt-out angekündigt.

Schlagzeilen machten auch Äußerungen des neuen Sejmmarschalls Radosław Sikorski in einem Interview für das amerikanische online-Nachrichtenmagazin „Politico«. Laut der Veröffentlichung hat der russische Präsident Wladimir Putin im Jahr 2008 Ministerpräsident Donald Tusk eine Aufteilung der Ukraine vorgeschlagen. Zwar ist Sikorski mit einer Entschuldigung zurückgerudert und auch Tusk sowie die russische Regierung haben dementiert, aber der Schaden war angerichtet und die Regierung hatte ihre erste innen- wie außenpolitische Krise. Allerdings stellte sich Ewa Kopacz nach einem Gespräch hinter den Sejmmarschall, so dass eine Abberufung, wie von der Opposition gefordert, keine Chance hat.

Eine weiter reichende Bewertung der Regierung ist momentan kaum möglich, da noch nicht einmal die sprichwörtlichen einhundert Tage verstrichen sind. Aber erste Personalentscheidungen und Umfragewerte erlauben vorsichtige Aussagen darüber, ob es sich um eine Übergangsregierung von Tusks Gnaden oder womöglich um ein dauerhafteres Projekt handelt.

\section{Regierung auf Abruf?}

Bereits in den ersten Kommentaren zur Personalie Kopacz wurde von manchen Kommentatoren und auch von Oppositionspolitikern gemutmaßt, dass Ewa Kopacz letztlich von Tusk abhängen und die Regierung noch von ihm gesteuert würde. Aber bereits in ihrem Exposé distanzierte sich Ewa Kopacz deutlich und unterstrich, dass sie die Regierungschefin sei und die volle Verantwortung übernehme. Auf einem Parteitag der PO Anfang Oktober 2014 sagte sie, dass sich die Partei von den Menschen entfernt habe und sie - wie im Exposé hervorgehoben - die Interessen der einfachen Wähler realisieren möchte. Ob ihre Regierung nur eine Regierung auf Abruf sein wird, wird im Wesentlichen von zwei Faktoren abhängen: Erstens davon, ob es ihr gelingen wird, die Bürgerplattform geschlossen hinter sich in den Wahlkampf zu führen, und zweitens ob sie imstande sein wird, über Regierungshandeln das Vertrauen der Menschen für einen weiteren Regierungsauftrag zu erlangen.

Was die Bürgerplattform betrifft, hat sich Ewa Kopacz nicht gescheut, prominente $P O$-Politiker mit großer Hausmacht in ihre Regierungsmannschaft aufzunehmen und so das Profil der $P O$ für die Wahlen zu schärfen und zugleich die Reihen zu schließen. Grabarczyk und Schetyna müssen sich nun in ihren Ressorts bewähren und dort Erfolge vorweisen. Konsequenterweise wird die Ministerpräsidentin auch Donald Tusk im Amt des Parteivorsitzenden auf einem Parteitag im November folgen. Allerdings soll sie die Partei nur bis zu den Wahlen im nächsten Jahr führen; erst dann ist eine längerfristige Bestimmung des Parteichefs vorgesehen.

Die von manchen Kritikern nach Vorstellung des Exposés im Sejm bemängelte fehlende Strategie könnte für die neue Ministerpräsidentin auch ein Vorteil sein. Wie der Soziologe und politische Kommentator Henry Domański in einem Interview hervorhob, erwarten die Polen heute konkrete Entscheidungen und keine Revolution. Zudem sind viele Menschen im Land die scharfen politischen Auseinandersetzungen und Grabenkämpfe leid, so dass der Appell von Ewa Kopacz, den Hass zu beenden, positiv aufgenommen werden dürfte. Da ihr zudem gerade einmal ein gutes Jahr zur Verfügung steht, um die Wähler zu überzeugen, dürfte es taktisch klug gewesen sein, schwierige normative Fragen zumindest im Exposé nicht in den Vordergrund gestellt zu haben. Weder die Frage der In-vitro-Befruchtung noch gleichgeschlechtliche Lebenspartnerschaften wurden erwähnt und auch der Landwirtschaft wurde kein Raum eingeräumt. Jedoch ist gerade hier nach wie vor großer Reformbedarf, weil das System der Landwirtschaftlichen Sozialversicherung (Kasa Rolniczych Ubezpieczeń Społecznych - KRUS) nach einem Urteil des Verfas- 
sungsgerichtes aus dem Jahr 2010 reformiert werden muss: Das Gericht war der Ansicht, dass die Landwirte ihre Abgaben für die Gesundheitsversicherung selbst aufbringen müssten. Allerdings regelt ein Gesetz aus dem Jahr 2004 bis heute die Finanzierung dieser Abgaben aus dem Staatssäckel. Da das Verfassungsgericht für eine entsprechende Gesetzesänderung 15 Monate Zeit ließ, ist eine neue Regelung mehr als überfällig. Allerdings ist auch hier für die neue Ministerpräsidentin wenig politischer Gewinn zu machen, da sich ihr Koalitionspartner, die Bauernpartei PSL, kaum leicht mit einer Änderung des Systems wird anfreunden können. Hier dürften Veränderungen erst nach der Wahl anstehen.

Punkten kann man als neuer Regierungschef eher auf der diplomatischen Bühne und hier spricht einiges dafür, dass die Beziehungen zu Deutschland weiterhin gut sein werden. Während des Energiegipfels der EU akzeptierte Kanzlerin Merkel die polnische Position, was Ewa Kopacz den Start auf europäischer Ebene erleichtert hat und für Rückenwind in Polen gesorgt haben dürfte. Am 20. November soll es überdies zu einem symbolträchtigen Treffen zwischen Angela Merkel und Ewa Kopacz in Kreisau (Krzyżowa) kommen, 25 Jahre nach der Versöhnungsgeste zwischen Helmut Kohl und Tadeusz Mazowiecki.

Letztlich wird aber der Wähler über den Erfolg der neuen Regierung entscheiden und nach den bisherigen Umfragen kann man Ewa Kopacz einen guten Start bescheinigen. Zwar liegen nach Daten des Meinungsforschungsinstitutes CBOS (Centrum Badania Opinii Społecznej - Zentrum zur Erforschung der öffentlichen Meinung) vom Oktober dieses Jahres $P O$ und $P i S$ ex aequo bei 34 Prozent in der traditionellen Sonntagsfrage, aber die Sympathiewerte für die neue Ministerpräsidentin sind nach oben geschossen. Noch im September bedachten 48 Prozent der Befragten die Sejmmarschallin mit Sympathie, etwas mehr als für den damaligen Ministerpräsidenten Tusk (46 Prozent) und etwas weniger als für den damaligen Außenminister Sikorski (49 Prozent). Nur Staatspräsident Komorowski lag mit 79 Prozent Zustimmung prominent an der Spitze. Im Oktober legte die frisch ernannte Ministerpräsidentin um 11 Punkte zu und befindet sich nun mit einem Vertrauenswert von 59 Prozent deutlich an zweiter Stelle hinter Staatspräsident Komorowski (80 Prozent).

Auch die an ihre Regierung geknüpften Erwartungen und Hoffnungen sind größer als beim zweiten Kabinett Tusk im Jahr 2011. Hier hatte sich der TuskEffekt bereits etwas verbraucht und seine Wahlerfolge sind auch der nach Ansicht vieler Wähler mangelnden Alternative zuzuschreiben. Allerdings hat die Regierung Kopacz nach den vorliegenden Daten nur wenig mehr Anhänger als Jarosław Kaczyński im Sommer 2006. Viele Menschen empfinden eine große Distanz gegenüber der Politik und damit auch gegenüber der neuen Regierung, wie die Regierungschefin ja selbst in ihrem Exposé unterstrich.

Ein durchschlagender Erfolg wäre ihre Regierung nicht nur, wenn sie und ihre Partei die nächsten Wahlen gewinnen würden, sondern vor allem, wenn es ihr gelänge, das Vertrauen in die Politik wieder zu vergrößern. Ein erster Test dafür werden die Kommunal- und Regionalwahlen am 16. November sein.

Über den Autor

Prof. Dr. Stefan Garsztecki, Politologe, ist Professor für Kultur- und Länderstudien Ostmitteleuropas an der TU Chemnitz. 


\title{
Exposé der Ministerpräsidentin Ewa Kopacz vom 1. Oktober 2014 (Auszüge aus dem Stenogramm)
}

\author{
Der Sejm, 1.10.2014 \\ Ewa Kopacz, Vorsitzende des Ministerrates
}

Herr Staatspräsident, Herr Sejmmarschall, Herr Senatsmarschall, sehr geehrte Damen und Herren Abgeordnete, sehr geehrte Damen und Herren,

ich stehe heute als neue Ministerpräsidentin der Republik Polen vor Ihnen. Das ist für mich eine große Ehre und Auszeichnung. Ich möchte mich heute in Ihrer Anwesenheit feierlich zum treuen Dienst an Polen und den Polen verpflichten und das Programm meiner Regierung vorstellen. Gewöhnlich findet ein Regierungswechsel infolge verlorener Wahlen statt oder er ist die Konsequenz einer ernsten politischen Krise. Heute ist das nicht der Fall. Heute resultiert der Regierungswechsel aus einem großen Erfolg unseres Landes, und zwar der Wahl von Donald Tusk zum Präsidenten des Europäischen Rates. Noch vor 25 Jahren träumten wir davon, dass Polen zum vereinigten Europa gehört und heute wird Donald Tusk sein Präsident. Als Polin bin ich auf diesen Erfolg stolz. Er wäre nicht möglich geworden ohne die rund sieben Jahre Regierung aus Bürgerplattform [Platforma Obywatelska - PO] und Polnischer Bauernpartei [Polskie Stronnictwo Ludowe - PSL]. Herr Vorsitzender, wir alle wünschen Ihnen Erfolg, alle. [...] Ich bin es, die an der Spitze der polnischen Regierung steht, dieser neuen Regierung und für sie übernehme ich die volle Verantwortung - natürlich, wenn dies das Hohe Haus beschließt. Ich danke Donald Tusk für die vergangenen sieben Jahre und möchte unterstreichen, dass der Erfolg Polens, den wir nach '89 erlangt haben, im politischen Sinne viele Väter hat. Alle meine Vorgänger im Amt des Ministerpräsidenten der Republik Polen trugen ihren Teil bei, auch die hier im Saal Anwesenden. Das ist das Wesen der Demokratie, dass die Wähler die Regierenden auswechseln. Aber die Wähler wählen auch die Opposition, das heißt diejenigen, deren Pflicht es ist, der Regierung auf die Finger zu schauen. Ich bin offen für Debatten und Kritik. Ich gestatte auch keinem meiner Minister, vor einer Debatte zu flüchten und auf Kritik nicht zu antworten. Aber bemühen wir uns [...], dass die Kritik und die Debatte nicht zu einer Art politischem Bürgerkrieg werden. Polen ist eins und es gehört gleichermaßen denen, die für die Regierung stimmen, wie auch denen, die ihre Stimme der Opposition geben. Heute freuen wir uns zu Recht und wissen die Errungenschaften der polnischen Freiheit und polnischen Demokratie zu schätzen. Aber wir dürfen nicht davor die Augen verschließen, dass sehr viele Polen nicht damit zufrieden sind, was in Polen geschieht. Sie haben das Gefühl, dass nicht alles gut läuft. Aber vor allem haben sie immer noch wenig Vertrauen in die Institutionen des öffentlichen Lebens. Das betrifft auch oder sogar vor allem dieses Hohe Haus. Deshalb will ich heute sehr klar sagen: Wir sind hier nicht um unserer selbst willen, sondern wir sind hier für die Polen. Meine Aufgabe, die wichtigste Aufgabe wird vor allem sein, das Vertrauen der Polen in die Regierung, die Politik und die Politiker zu vergrößern und aufzubauen. Ich weiß, dass das eine sehr ehrgeizige Aufgabe ist, aber ich glaube, sie ist realistisch. Der erste Schritt zu ihrer Verwirklichung ist das beständige Gespräch mit den Menschen und die Anhörung ihrer Probleme.

$[\ldots]$

Meine Damen und Herren Abgeordnete, das Vertrauen der Bürger aufzubauen erfordert von uns auch, eine überparteiliche Verständigung in den Angelegenheiten zu erreichen, die heute polnische Staatsräson sind, also in der polnischen Außen- und Verteidigungspolitik. Wir müssen uns gemeinsam um Sicherheit und Respekt für Polen in Europa, aber auch in der Welt kümmern. Das erfordert eine erweiterte Debatte in diesem Saal, auch mit der Opposition, deren Unterstützung wesentlich sein wird; deshalb wird der Chef des Außenressorts bis Ende Oktober Auskunft über die Außenpolitik geben. Jede Außenpolitik muss sich auf ein Wertesystem stützen. Diese Werte sind nur so stark, wie der Wille und die Entschlossenheit, sie zu verteidigen. Die Ereignisse in der Ukraine machen uns heute sehr deutlich, wie wichtig angesichts des Autoritarismus und des Chaos, der zwei Plagen der gegenwärtigen Welt, die Solidarität der demokratischen Staaten ist. Gleichzeitig zeigt die ukrainische Krise, wie wesentlich es ist, dass wir nicht allein sind, wenn wir uns an dieser Herausforderung messen müssen, weil die Europäische Union und die NATO hinter uns stehen. Die Aufgabe meiner Regierung wird es sein, sich um Einheit und Solidarität des demokratischen Lagers zu bemühen. Das ist die wichtigste Staatsräson, damit es nicht zu einer Spaltung der Haltung des Westens kommt, dessen Teil wir sind, aber gleichermaßen wesentlich ist es, dass es nicht dazu kommt, dass Polen vereinsamt, weil wir uns unrealitsiche Ziele gestellt haben. Deshalb wird es auch ein Ziel meiner Regierung sein, eine pragmatische Politik gegenüber dem, was in der Ukraine geschieht, zu betreiben. Für Polen und seine Bürger hat die Beendigung der Kämpfe in der Ukraine und die Konsolidierung des ukrainischen Staates Schlüsselbedeutung. Wir hoffen, dass die letzten Gespräche 
über die Einstellung der Kämpfe Erfolge zeitigen. Das bedeutet nicht, und das unterstreiche ich mit allem Nachdruck, das bedeutet weder ein Einverständnis mit der Aneignung von Territorium des souveränen ukrainischen Staates noch die Akzeptanz der Grenzänderung in Europa mithilfe von Gewalt. Das ist eine Frage der fundamentalen Prinzipien der Welt, zu der wir gehören. Wir unterstützen die proeuropäische Richtung der Entwicklung der Ukraine, aber wir werden nicht die Ukrainer vertreten, auf denen die Last der Verantwortung liegt, ihr Land zu reformieren. Ich werde mich dafür einsetzen, das Engegement der Ukrainer für die Freiheit auf allen Feldern zu unterstützen. Wir wollen der Ukraine bei der wirtschaftlichen, strukturellen, militärischen und politischen Transformation helfen.

Meine Damen und Herren Abgeordnete, eines der wichtigsten Ziele meiner Regierung wird die weitere Stärkung unserer Position in der Europäischen Union sein. Dank seiner vorhersehbaren und glaubwürdigen Politik der letzten Jahre kam Polen endlich in die erste europäische Liga und begann die Rolle zu entdecken, die seinem Potential und seinen Ambitionen entspricht. Unser Land wird sich darum bemühen, dass die Europäische Union die Idee der Energiesolidarität umsetzt. Deshalb müssen wir auch den Aufbau der Energieunion aktiv unterstützen, die von meinem Vorgänger vorgeschlagen wurde und heute von der deutlichen Mehrheit der europäischen Führer unterstützt wird. Man darf sich nicht mit der Situation abfinden, dass Polen und andere Staaten der Region auf die Gnade oder Ungnade externer Gaslieferer angewiesen sind, die ihre Rohstofflieferungen oder -preise von aktuellen politischen Zielen abhängig machen. Meine Regierung wird daher die Europäische Union davon überzeugen, jeglichen monopolistischen Praktiken und Preismanipulationen wirksam ein Ende zu setzen. Wir sind uns dessen bewusst, wie wichtig Umweltschutz ist. Auf dem Gipfel des Europäischen Rates im Oktober wird das Projekt neuer Ziele zur Reduktion der Gasemissionen geprüft. Meine Regierung wird keinen Vorschriften zustimmen, die zusätzliche Kosten für unsere Wirtschaft sowie höhere Energiepreise für die Konsumenten bedeuten. Meine Damen und Herren Abgeordnete, unser Land wird sich eindringlich um die schnellstmögliche Unterzeichnung des Freihandelsabkommens zwischen der Europäischen Union und den Vereinigten Staaten bemühen. Es wird die weltweit größte Freihandelszone schaffen und enormen Einfluss auf ein gutes Wirtschaftsklima nehmen, womit vertiefte politische Beziehungen zwischen Europa und Amerika einhergehen. Angesichts der Geschehnisse in der Ukraine wird die Intensivierung der Beziehungen mit den Vereinigten Staaten umso wichtiger. Deshalb wird meine Regierung alle Bemühungen unternehmen, dass die Vereinigten Staaten ihre militärische Anwesenheit in Polen verstärken werden.

Meine Damen und Herren Abgeordnete, die polnischen Familien unterhalten sich seit einigen Monaten darüber, ob unser Land sicher sei. Unsere tragische Geschichte ruft solche grundsätzlichen Fragen insbesondere jetzt hervor, da an unserer Grenze der seit Jahrzehnten größte bewaffnete Konflikt ausgetragen wird. Meine Regierung wird Polen und den Polen Sicherheit garantieren. Um dieses Ziel zu verwirklichen, werden die Verteidigungsausgaben ab 2016 auf zwei Prozent des Bruttoinlandsprodukts erhöht, das stellt zusätzliche 800 Millionen Zloty für eine moderne Ausrüstung für die Polnische Armee im Jahr 2016 sicher und entsprechend mehr in den folgenden Jahren. Wenn wir unsere Armee modernisieren, werden wir das innovative Potential der polnischen Rüstungsindustrie aufbauen, die sich als Polnische Rüstungsgruppe konsolidiert. Das Fundament unserer Verteidigung sind die Soldaten, die sich bei ihrem verantwortungsvollen und schwierigen Dienst der großen Unterstützung der Bevölkerung erfreuen. Meine Regierung wird sich um ihre Lage kümmern. Ab Anfang 2015 werden wir zusätzliche 39 Millionen Zloty für die Soldaten zur Verfügung stellen und die längere Dienstzeit der Offiziere und Unteroffiziere prämieren.

[...]

Die schwierige geopolitische Lage im Zusammenhang mit dem Konflikt im Osten trifft die polnischen Unternehmer. Das russische Embargo für polnische Produkte bedroht die Stabilität unserer Firmen und der in ihnen Beschäftigten. Deshalb bereiten wir ein Antikrisengesetz vor, dank dessen diejenigen Firmen, die wegen des Embargos Verluste erleiden, Zuzahlungen zu den Gehältern oder Fortbildungen für Angestellte in Anspruch nehmen können oder eine Erstattung der Sozialversicherungsbeiträge. Wir werden in den Gesetzesentwurf die Möglichkeit schreiben, im Rahmen dieser Hilfe bis zu 500 Millionen Zloty aus dem Fonds für Garantierte Arbeitsleistungen zu erhalten. Das russische Embargo für polnische Lebensmittel betraf viele landwirtschaftliche Produzenten. Wir haben das Recht, von der Europäischen Union zu erwarten, dass ihre Hilfe für den ländlichen Raum in diesem Bereich den Verlusten, die wir tragen, angemessen sein wird. Ich will klar und ausdrücklich feststellen - die bisherigen Vorschläge der Europäischen Kommission stellen uns nicht zufrieden. Wir erwarten, dass der EU-Kommissar für Landwirtschaft den polnischen Erwartungen entgegen kommt. Gleichzeitig werden wir bis zum Jahresende einen Fonds zur Stabilisierung der Einkommen in der Landwirtschaft einführen, der sehr wichtig ist in Hinblick auf die immer häufigeren Fälle, dass termingerechte Zahlungen an die Landwirte für gelieferte Waren nicht eingehalten werden oder dass die Preise drastisch fallen. Der Fonds entsteht aus Abschreibungen in Höhe von 0,2 Prozent des Wertes der verkauften Produkte. 
Meine Damen und Herren Abgeordnete, in Polen hat die Kohle strategische Bedeutung. Eine der ersten Entscheidungen, die ich getroffen habe, ist die Beschleunigung der Arbeiten an Gesetzen, die in vernünftiger Weise drei grundlegende Ziele im Rahmen der Energiesicherheit Polens miteinander verbinden. Erstens müssen wir den polnischen Bergbau vor unehrlicher Konkurrenz schützen. Zweitens werde ich keine Mühe scheuen, damit die Branche endlich rentabel arbeitet. Zu diesem Ziel führen Modernisierung und Umstrukturierung. Drittens muss ich die Sicherheit einer jeden polnischen Familie berücksichtigen. Die polnischen Wohnungen müssen beheizt werden und die Energiekosten dürfen nicht die Haushaltskasse ruinieren. Die entworfenen Regulierungen, die wir schnell auf den parlamentarischen Weg gebracht haben, sehen konzessionierte Verkäufe vor, sie benennen die Qualitätsanforderungen an die Kohle und die Änderungen im Gesetz für öffentliche Ausschreibungen, die Produkte aus der Union bevorzugen. Wir werden auch die Entwicklung moderner Kohletechnologien unterstützen. Die Zeit, die wir dank der Einführung dieser Regulierungen gewinnen, nutzen wir, um die Kosten für den Bergwerksbetrieb so zu senken, dass die polnische Kohle auf dem Markt konkurrenzfähig ist.

Meine Damen und Herren Abgeordnete, eine der schönsten Ideen, die Polen der Welt mitgab, ist die Idee der Solidarität. Diese Solidarität in jeder Lage, in jeder Generation, zu jeder Zeit müssen wir aufs Neue definieren. Heute möchte ich vor Ihnen über die Solidarität zwischen den Generationen sprechen, die Polen im 21. Jahrhundert braucht. Die jüngeren Generationen dürfen dem Schicksal der älteren Generationen nicht gleichgültig gegenüber stehen und umgekehrt. Eine sichere Zukunft Polens bedeutet, dass wir die Herausforderung der demographischen Krise annehmen müssen. Die Geburt eines Kindes und ein Kind zu haben müssen die besondere Unterstützung unseres Staates genießen. Deshalb bestimmt meine Regierung zusätzliche Mittel für den Bau von Krippen und Kindergärten. 2015 werden wir die Summe für den Bau von Krippenplätzen von 50 Millionen auf 100 Millionen erhöhen. In den Jahren 2015 bis 2020 werden wir mit Hilfe von Geldern aus dem Europäischen Sozialfonds und einer speziellen Steuerermäßigung [...] die Gründung von Betriebskrippen und -kindergärten unterstützen. Für dieses Ziel stellen wir über zwei Milliarden Zloty bereit. Wir wollen, dass ab 2016 alle Eltern den Elternurlaub nutzen können, die bisher diese Möglichkeit nicht hatten. Das heißt: Eltern, die arbeitslos sind, die auf Werkvertragsbasis arbeiten, Studierende und Landwirte. Sie erhalten eine neue Elternleistung, die sie während der zwölf Monaten nach der Geburt des Kindes beziehen, bei Zwillingen, Drillingen und mehr Kindern entsprechend länger. Die Leistung beträgt zirka 1.000 Zloty. Die Familienpolitik des Staates sollte die Eltern ermuntern, arbeiten zu gehen, und es ihnen nicht verleiden. Es darf nicht sein, dass sie keine Arbeit annehmen, weil dies den Verlust ihrer staatlichen Leistungen bedeuten würde. [...]

Meine Damen und Herren Abgeordnete, viele junge Menschen, mit denen ich spreche, signalisierten mir ein ernstzunehmendes Problem, auf das sie stoßen, wenn sie gleich nach dem Studium in den Arbeitsmarkt eintreten. Die Erwartungen der Arbeitgeber an die jungen Hochschulabsolventen betreffen sehr häufig ihre praktische Berufserfahrung. Ich möchte, dass die öffentliche Verwaltung hilft, dieses Problem zu lösen und schlage daher ein Praktikumsprogramm für Studierende in den Ämtern der öffentlichen Verwaltung vor. So werden Studierende Praxiserfahrungen machen und die Hochschulen nicht nur reicher an Wissen, sondern auch an praktischen Erfahrungen verlassen. Es ist prima, wenn ein junger Mensch aus Polen die Möglichkeit bekommt, im Ausland zu studieren. Aber es ist sehr schlecht, wenn er im Ausland bleibt und seine Talente und sein Wissen nicht der Entwicklung des Vaterlands dienen. Meine Regierung schlägt jungen Polen, den begabtesten, vor, dass sie ab dem Jahr 2016 die Möglichkeit haben werden, an den besten ausländischen Universitäten zu studieren, wobei diese Möglichkeit vom polnischen Staat finanziert wird. Unter einer Bedingung: Jeder dieser jungen Menschen wird mindestens fünf Jahre nach der Rückkehr vom Studium in unserem Land arbeiten müssen. An dieser Stelle möchte ich mich dazu verpflichten, dass wir die Entwicklung der polnischen Wissenschaft unterstützen. Wir werden ihre Finanzierung auf zwei Prozent des Bruttoinlandsprodukts bis zum Jahr 2020 anheben. Wenn ich einerseits an die Erfolge im Hochschulwesen im freien Polen erinnere, muss ich andererseits allerdings mit Bedauern feststellen, dass wir in den letzten Jahren dem Berufsschulwesen zu wenig Aufmerksamkeit geschenkt haben. Berufsschulen, die an die Bedürfnisse des Marktes angepasst sind, sind eine Garantie dafür, dass der polnischen Wirtschaft nicht die Facharbeiter fehlen werden. Deshalb wird der Bildungsminister noch in diesem Jahr beginnen, ein Programm für den Aufbau des Berufsschulwesens in Zusammenarbeit mit den Unternehmern umzusetzen. Dann erhalten junge Menschen eine solide allgemeine Ausbildung, konkrete Qualifikationen, und es wird ihnen auch leichter fallen, eine Arbeit nach Beendigung der Ausbildung zu finden.

Meine Damen und Herren, ich bin stolz, dass in der polnischen Tradition die Sorge um ältere Menschen, ihre Gesundheit und Sicherheit einen so wichtigen Platz einnimmt. Heutzutage haben viele Berufstätige Angst um das Schicksal ihrer Eltern und Großeltern. Die Alltagssorgen und der Beruf verursachen sehr häufig, dass diese Menschen ihren Nächsten im fortgeschrittenen Alter nicht so viel Zeit widmen können, wie sie gern wollen. Ich möchte in Polen die in entwickelten Ländern des Westens bekannte Institution der Tagespflegeeinrichtungen gründen, in 
denen die Senioren medizinische Hilfe, interessante Beschäftigungsangebote und das Gefühl von Sicherheit erhalten. Das Geld für die Einrichtung solcher Häuser finden wir in den europäischen Finanzmittelpools. Die Kosten für den Aufenthalt der Senioren in diesen Einrichtungen würde zu einem Drittel der Staat decken, zu einem Drittel die territoriale Selbstverwaltung und zu einem Drittel die betreffende Privatperson, sie selbst oder ihr Betreuer. An dieser Stelle möchte ich die Verpflichtung gegenüber den Senioren bekräftigen, dass die Regierung ihr Versprechen einlösen wird, die Renten anzuheben, das hier in diesem Saal von meinem Vorgänger Donald Tusk gemacht wurde. Im Jahr 2015 werden wir 3,8 Milliarden Zloty für dieses Ziel bestimmen. Mit Blick auf die Senioren planen wir auch die Gründung eines Instituts für Geriatrie, als spezielle Einrichtung für die Heilung und Betreuung älterer Menschen. Die Gründung einer solchen Institution ermöglicht uns, eine größere Anzahl von Geriatern auszubilden, die heute in Polen in dramatischem Ausmaß fehlen.

$[\ldots]$

Meine Damen und Herren Abgeordnete, das sichtbarste Beispiel für den Erfolg Polens der letzten Jahre sind die großen Infrastrukturinvestitionen. Sie verändern das Bild unseres Landes, aber sie verändern auch unser Leben, verbessern den Komfort und die Sicherheit. Im Jahr 2014 geben wir über 160 Kilometer Schnellstraßen zur Nutzung frei und unterzeichnen Verträge für den Bau weiterer 300 Kilometer. In den nächsten Wochen werden wir schon über die Schnellstraße S8 von Breslau nach Sieradz fahren. Bis Ende 2015 werden 250 Kilometer Schnellstraßen freigegeben und Verträge über weitere 500 Kilometer unterzeichnet. In den Jahren 2014 bis 2020 werden wir in Polen 1.770 Kilometer Autobahn sowie Schnellstraßen und 35 Umgehungsstraßen für knapp 93 Milliarden Zloty bauen. Wir werden ein komplettes Autobahnnetz zur Nutzung freigeben: die A1, A2 und A4 und Schnellstraßen, hier seien nur die S3, S5, S6, S7, S8 und S17 genannt und die komplette Umgehungsstraße um Warschau herum.

$[\ldots]$

Meine Damen und Herren Abgeordnete, es ist eine Sache der Gerechtigkeit, dass die Kultur allen gesellschaftlichen Gruppen unabhängig vom Wohnort und Lebensstandard zugänglich ist. Deshalb planen wir, das Projekt "Zugängliche Kultur« ins Leben zu rufen, dank dessen es günstigere Eintrittskarten für die wichtigsten Museen und Theater, kostenlose Kurse in kulturellen Einrichtungen für Kinder und Jugendliche und spezielle Angebote für Senioren geben wird. Wir werden für ein reichhaltigeres Angebot in den Bibliotheken sorgen und den kostenfreien Zugang über das Internet zu Büchern, Konzert- und Schauspielaufnahmen, auf denen Autorenrechte liegen, erweitern. Wir kreieren ein spezielles Bildungsprogramm, dessen besondere Aufmerksamkeit den historischen Orten und Museen gelten wird, die die Quelle für den Aufbau eines modernen Patriotismus und historischen Bewusstseins der jungen Generation sind.

Meine Damen und Herren Abgeordnete, ich bitte Sie heute nicht um einhundert Tage Ruhe, denn es geht hier nicht um Ruhe, sondern um die Möglichkeiten der Zusammenarbeit in den für Polen wichtigsten Angelegenheiten. Gerade deshalb bitte ich heute alle hier Anwesenden, und insbesondere die Opposition, um einhundert Tage Zusammenarbeit.

Ich glaube, dass es besonders bei solchen Angelegenheiten wie nationale Verteidigung, Energiesicherheit und die Zukunft der polnischen Kohle gelingen wird, in diesem Saal ein Einvernehmen erreichen. Gerade das erwarten alle Polen heute von uns und deshalb müssen wir es machen. In diesem Zusammenhang bitte ich das Hohe Haus, meinem Kabinett das Vertrauen auszusprechen. Vielen Dank.

Übersetzung aus dem Polnischen: Silke Plate

Quelle: 〈https://www.premier.gov.pl/expose-premier-ewy-kopacz-stenogram.html >

(abgerufen am 27.10.2014). 


\section{Einstellungen zur Regierung von Ewa Kopacz und zu Vorgängerregierungen}

Grafik 1: Wen würden Sie wählen, wenn am nächsten Sonntag Parlamentswahl wäre? (\%)

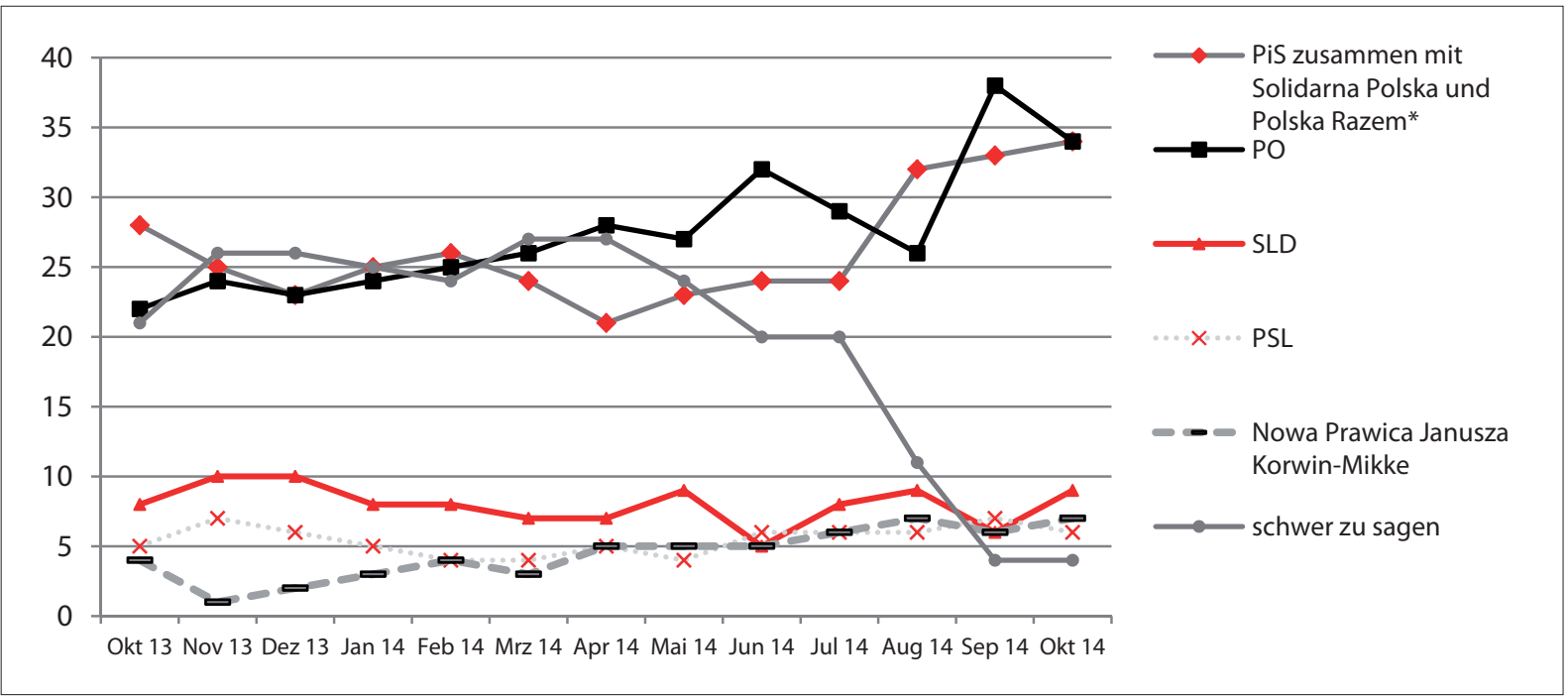

Anm.: Berücksichtigt wurden die Antworten derer, die angaben, zur Wabl gehen zu wollen.

* Unterstützung für PiS: Oktober 2013 - Juli 2014

PiS-Prawo i Sprawiedliwośc/Recht und Gerechtigkeit; Solidarna Polska Zbigniewa Ziobra - Solidarisches Polen von Zbigniew Ziobro; Polska Razem Jarosława Gowina - Polen Gemeinsam von Jarostaw Gowin; PO - Platforma Obywatelska/Bürgerplattform; SLD Sojusz Lewicy Demokratycznej/Demokratische Linksallianz; PSL - Polskie Stronnictwo Ludowe/Polnische Bauernpartei; Nowa Prawica Janusza Korwin-Mikke - Neue Rechte von Janusz Korwin-Mikke

Quelle: CBOS Nr 140/2014 Preferencje partyjne w październiku [Parteipräferenzen im Oktober]. Warszawa 10/2014. <www.cbos.pl>

Tabelle 1: Wen würden Sie wählen, wenn am nächsten Sonntag Parlamentswahl wäre? (\%)

\begin{tabular}{|c|c|c|c|c|c|c|c|c|c|c|c|c|c|}
\hline & $\begin{array}{l}\text { Okt. } \\
2013\end{array}$ & $\begin{array}{l}\text { Nov. } \\
2013\end{array}$ & $\begin{array}{l}\text { Dez. } \\
2013\end{array}$ & $\begin{array}{l}\text { Jan. } \\
2014\end{array}$ & $\begin{array}{l}\text { Feb. } \\
2014\end{array}$ & $\begin{array}{l}\text { März } \\
2014\end{array}$ & $\begin{array}{l}\text { April } \\
2014\end{array}$ & $\begin{array}{l}\text { Mai } \\
2014\end{array}$ & $\begin{array}{l}\text { Juni } \\
2014\end{array}$ & $\begin{array}{c}\text { Juli } \\
2014\end{array}$ & $\begin{array}{l}\text { Aug. } \\
2014\end{array}$ & $\begin{array}{l}\text { Sept. } \\
2014\end{array}$ & $\begin{array}{l}\text { Okt. } \\
2014\end{array}$ \\
\hline $\begin{array}{l}\text { PiS zusammen } \\
\text { mit Solidarna } \\
\text { Polska und } \\
\text { Polska Razem }\end{array}$ & $28^{*}$ & $25^{*}$ & $23^{*}$ & $25^{*}$ & $26^{*}$ & $24^{*}$ & $21^{*}$ & $23^{*}$ & $24^{*}$ & $24^{*}$ & 32 & 33 & 34 \\
\hline $\mathrm{PO}$ & 22 & 24 & 23 & 24 & 25 & 26 & 28 & 27 & 32 & 29 & 26 & 38 & 34 \\
\hline SLD & 8 & 10 & 10 & 8 & 8 & 7 & 7 & 9 & 5 & 8 & 9 & 6 & 9 \\
\hline $\begin{array}{l}\text { Nowa Prawica } \\
\text { Janusza Kor- } \\
\text { win-Mikke }\end{array}$ & 4 & 1 & 2 & 3 & 4 & 3 & 5 & 5 & 5 & 6 & 7 & 6 & 7 \\
\hline PSL & 5 & 7 & 6 & 5 & 4 & 4 & 5 & 4 & 6 & 6 & 6 & 7 & 6 \\
\hline $\begin{array}{l}\text { Twój Ruch/ } \\
\text { Ruch Palikota }\end{array}$ & 4 & 3 & 3 & 3 & 3 & 3 & 1 & 3 & 1 & 2 & 4 & 2 & 2 \\
\hline $\begin{array}{l}\text { Solidarna } \\
\text { Polska } \\
\text { Zigniewa } \\
\text { Ziobra }\end{array}$ & 2 & 1 & 2 & 2 & 2 & 2 & 1 & 1 & 3 & 1 & - & - & - \\
\hline
\end{tabular}


Tabelle 1: Wen würden Sie wählen, wenn am nächsten Sonntag Parlamentswahl wäre? (\%) (Fortsetzung)

\begin{tabular}{|c|c|c|c|c|c|c|c|c|c|c|c|c|c|}
\hline & $\begin{array}{l}\text { Okt. } \\
2013\end{array}$ & $\begin{array}{l}\text { Nov. } \\
2013\end{array}$ & $\begin{array}{l}\text { Dez. } \\
2013\end{array}$ & $\begin{array}{l}\text { Jan. } \\
2014\end{array}$ & $\begin{array}{l}\text { Feb. } \\
2014\end{array}$ & $\begin{array}{l}\text { März } \\
2014\end{array}$ & $\begin{array}{l}\text { April } \\
2014\end{array}$ & $\begin{array}{l}\text { Mai } \\
2014\end{array}$ & $\begin{array}{l}\text { Juni } \\
2014\end{array}$ & $\begin{array}{c}\text { Juli } \\
2014\end{array}$ & $\begin{array}{l}\text { Aug. } \\
2014\end{array}$ & $\begin{array}{l}\text { Sept. } \\
2014\end{array}$ & $\begin{array}{l}\text { Okt. } \\
2014\end{array}$ \\
\hline $\begin{array}{l}\text { PJN/Pol- } \\
\text { ska Razem } \\
\text { Jarosława } \\
\text { Gowina }\end{array}$ & $0 * *$ & $1^{* *}$ & $1^{* *}$ & 3 & 2 & 2 & 3 & 2 & 1 & 2 & - & - & - \\
\hline $\begin{array}{l}\text { PPP - Sierpień } \\
\text { ' } 80\end{array}$ & 1 & 0 & 1 & 1 & 0 & 0 & 0 & 0 & 0 & 1 & 0 & 1 & 2 \\
\hline $\begin{array}{l}\text { Prawica } \\
\text { Rzeczypos- } \\
\text { politej }\end{array}$ & 0 & 0 & 0 & 0 & 1 & 0 & 0 & 0 & 0 & 0 & 1 & 0 & 0 \\
\hline andere & 3 & 2 & 2 & 1 & 2 & 2 & 2 & 2 & 3 & 2 & 3 & 2 & 3 \\
\hline $\begin{array}{l}\text { schwer zu } \\
\text { sagen }\end{array}$ & 21 & 26 & 26 & 25 & 24 & 27 & 27 & 24 & 20 & 20 & 11 & 4 & 4 \\
\hline
\end{tabular}

Anm.: Berücksichtigt wurden die Antworten derer, die angaben, zur Wahl gehen zu wollen.

*Unterstützung für PiS

**Unterstützung für PJN

PiS-Prawo i SprawiedliwośćlRecht und Gerechtigkeit; Solidarna Polska Zbigniewa Ziobra-Solidarisches Polen von Zbigniew Ziobro; Polska Razem Jarostawa Gowina - Polen Gemeinsam von Jarosław Gowin; PO - Platforma ObywatelskalBürgerplattform; SLD _ Sojusz Lewicy Demokratycznej/Demokratische Linksallianz; Nowa Prawica Janusza Korwin-Mikkego - Neue Rechte von Janusz Korwin-Mikke; PSL - Polskie Stronnictwo Ludowe/Polnische Bauernpartei; Ruch Palikota/Palikot-Bewegung; PJN - Polska Jest Najwazniejsza/Polen ist am Wichtigsten; PPP - Polska Partia Pracy Sierpień '80/Polnische Arbeitspartei-August '80; Prawica Rzeczypospolitej-Rechte der Republik

Quelle: CBOS Nr 140/2014 Preferencje partyjne w październiku [Parteipräferenzen im Oktober]. Warszawa 10/2014. <www.cbos.pl>

\section{Grafik 2: Die Bewertung der Tätigkeit des Sejm (\%)}

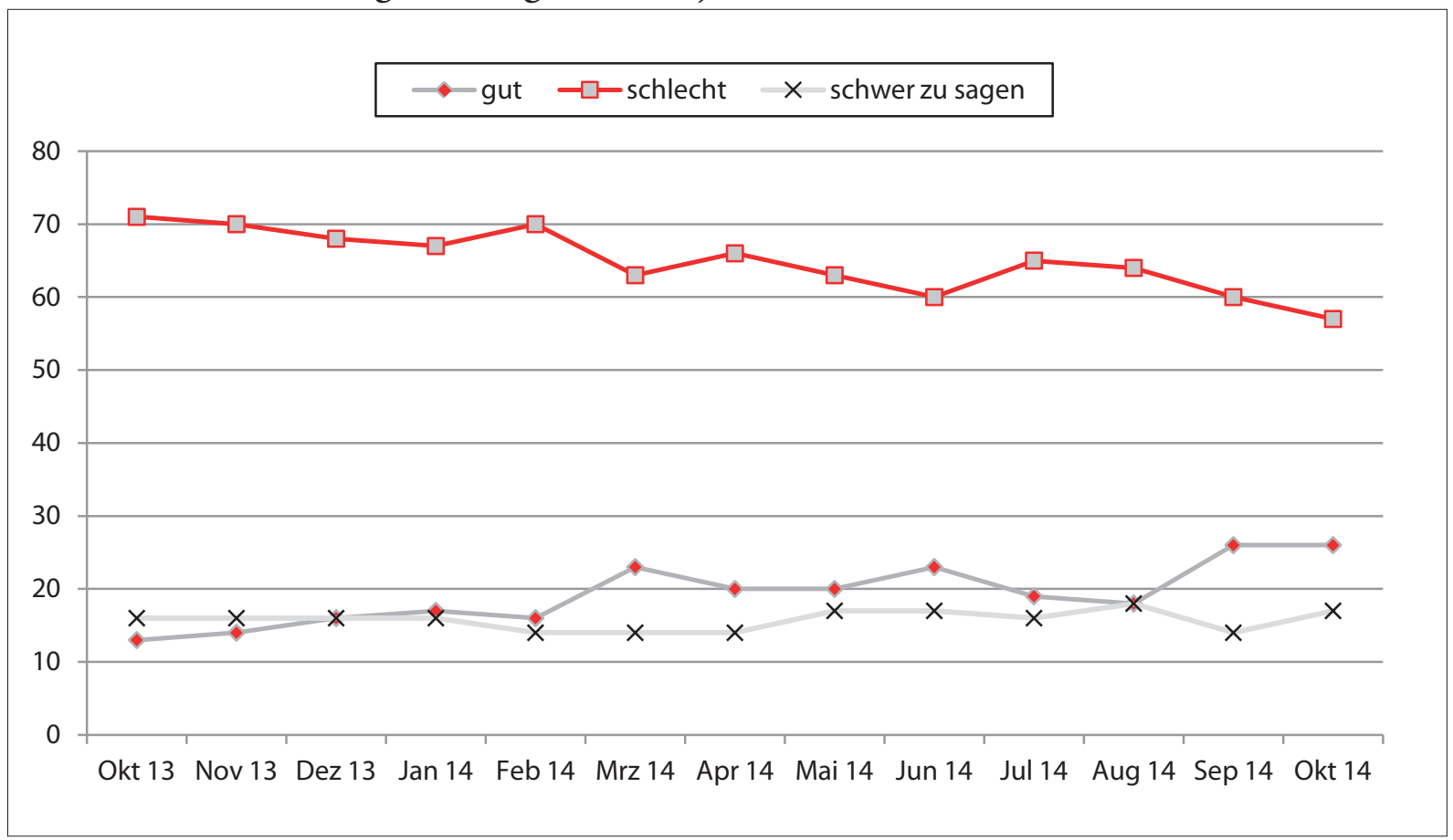

Quelle: CBOS Nr 141/2014 Oceny instytucji publicznych [Beurteilungen öffentlicher Institutionen]. Warszawa 10/2014. <www.cbos.pl> 
Grafik 3: Die Bewertung der Tätigkeit des Senats (\%)

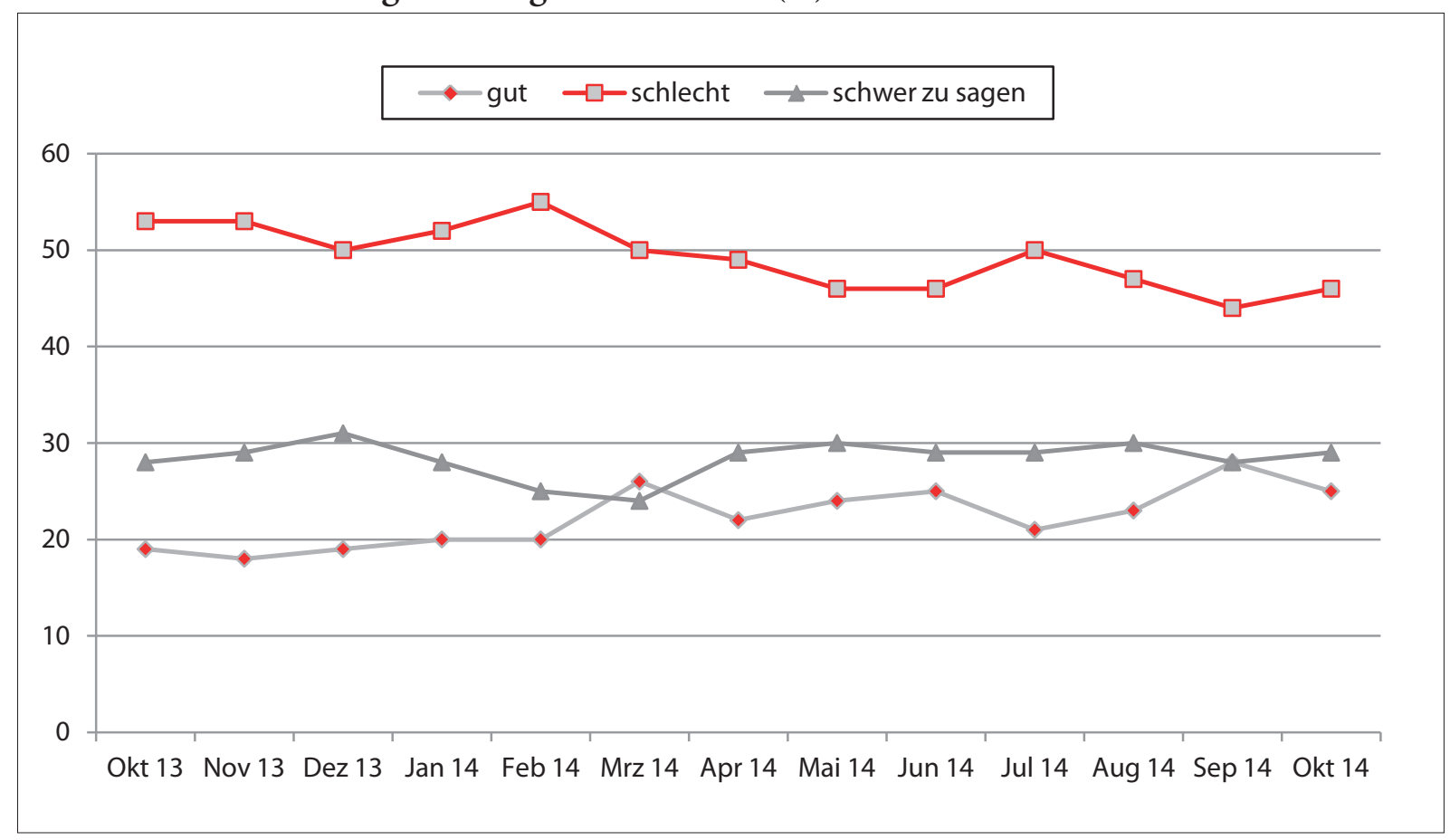

Quelle: CBOS Nr 141/2014 Oceny instytucji publicznych [Beurteilungen öffentlicher Institutionen]. Warszawa 10/2014. <www.cbos.pl>

Tabelle 2: Weckt die Regierung von Ewa Kopacz bei Ihnen...? (\%)

\begin{tabular}{|l|c|c|c|c|c|}
\hline $\begin{array}{c}\text { potentielle } \\
\text { Wählerschaft } \\
\text { von }\end{array}$ & $\begin{array}{c}\text { Hoffnung, } \\
\text { Zuversicht, dass } \\
\text { es besser wird }\end{array}$ & $\begin{array}{c}\text { Enttäuschung, } \\
\text { weil sich nichts } \\
\text { ändern wird }\end{array}$ & $\begin{array}{c}\text { Angst, dass es } \\
\text { schlechter wird }\end{array}$ & $\begin{array}{c}\text { Gleichgültig- } \\
\text { keit; interessiert } \\
\text { nicht }\end{array}$ & schwer zu sagen \\
\hline PO & 59 & 14 & 4 & 14 & 9 \\
\hline SLD & 56 & 17 & 10 & 13 & 4 \\
\hline PSL* & 50 & 19 & 11 & 15 & 5 \\
PiS & 28 & 22 & 25 & 18 & 7 \\
\hline $\begin{array}{l}\text { Nowa Prawica } \\
\text { Janusza Korwi- } \\
\text { na-Mikke* }\end{array}$ & 21 & 35 & 37 & 7 & 0 \\
\hline
\end{tabular}

*Die Angaben sind aufgrund der geringen Anzabl in der befragten Gruppe mit Vorsicht zu behandeln.

PO/Platforma Obywatelska - Bürgerplattform

SLD/Sojusz Lewicy Demokratycznej - Demokratische Linksallianz

PSL/Polskie Stronnictwo Ludowe - Polnische Banernpartei

PiS/Prawo i Sprawiedliwość-Recht und Gerechtigkeit

Nowa Prawica Janusza Korwina-Mikke - Neue Rechte von Janusz Korwin-Mikke

Quelle: CBOS Nr 145/2014 Pierwsze notowania rzadu Ewy Kopacz [Erste Umfragen zur Regierung von Ewa Kopacz]. Warszawa 10/2014. <www.cbos.pl> 


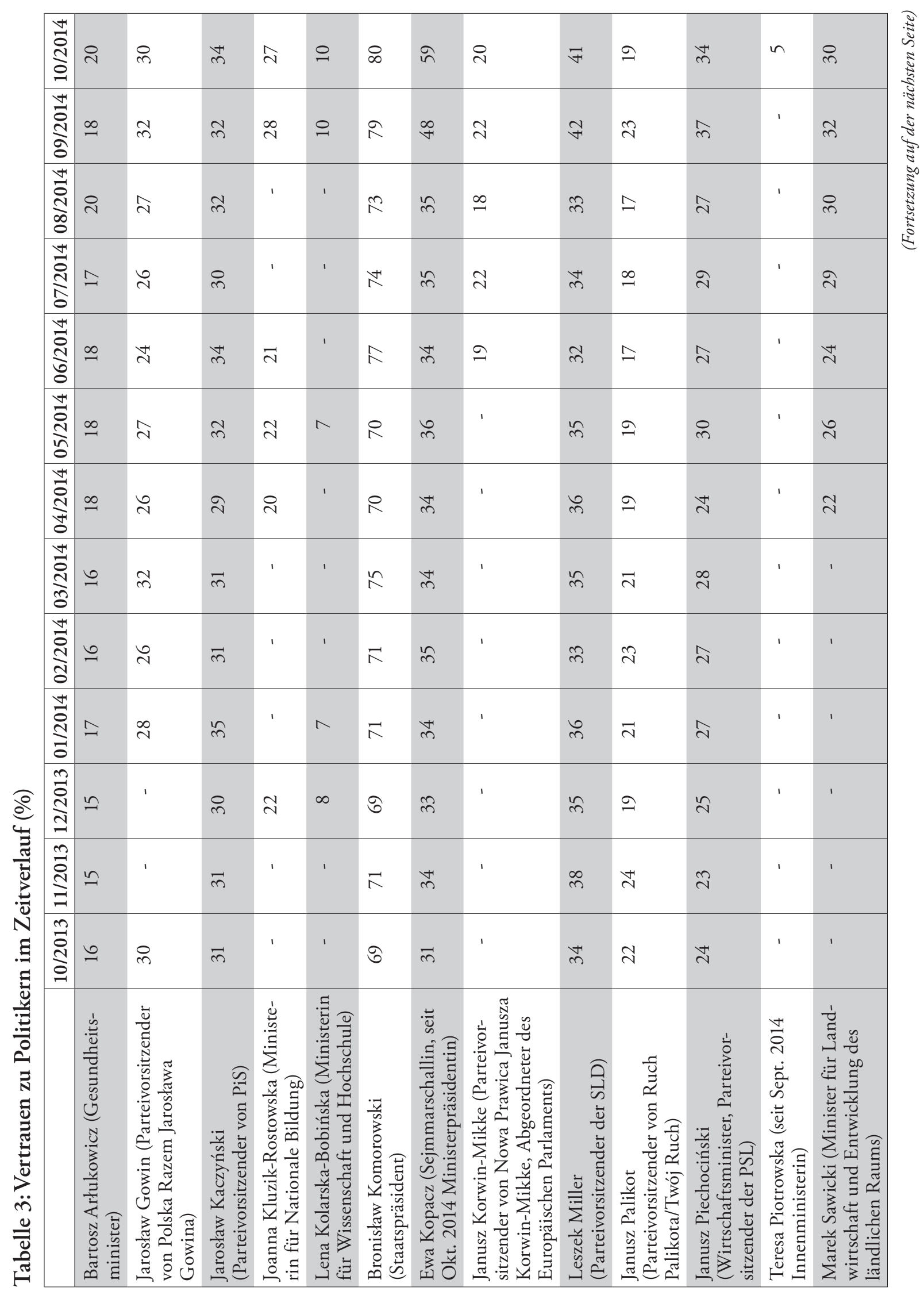




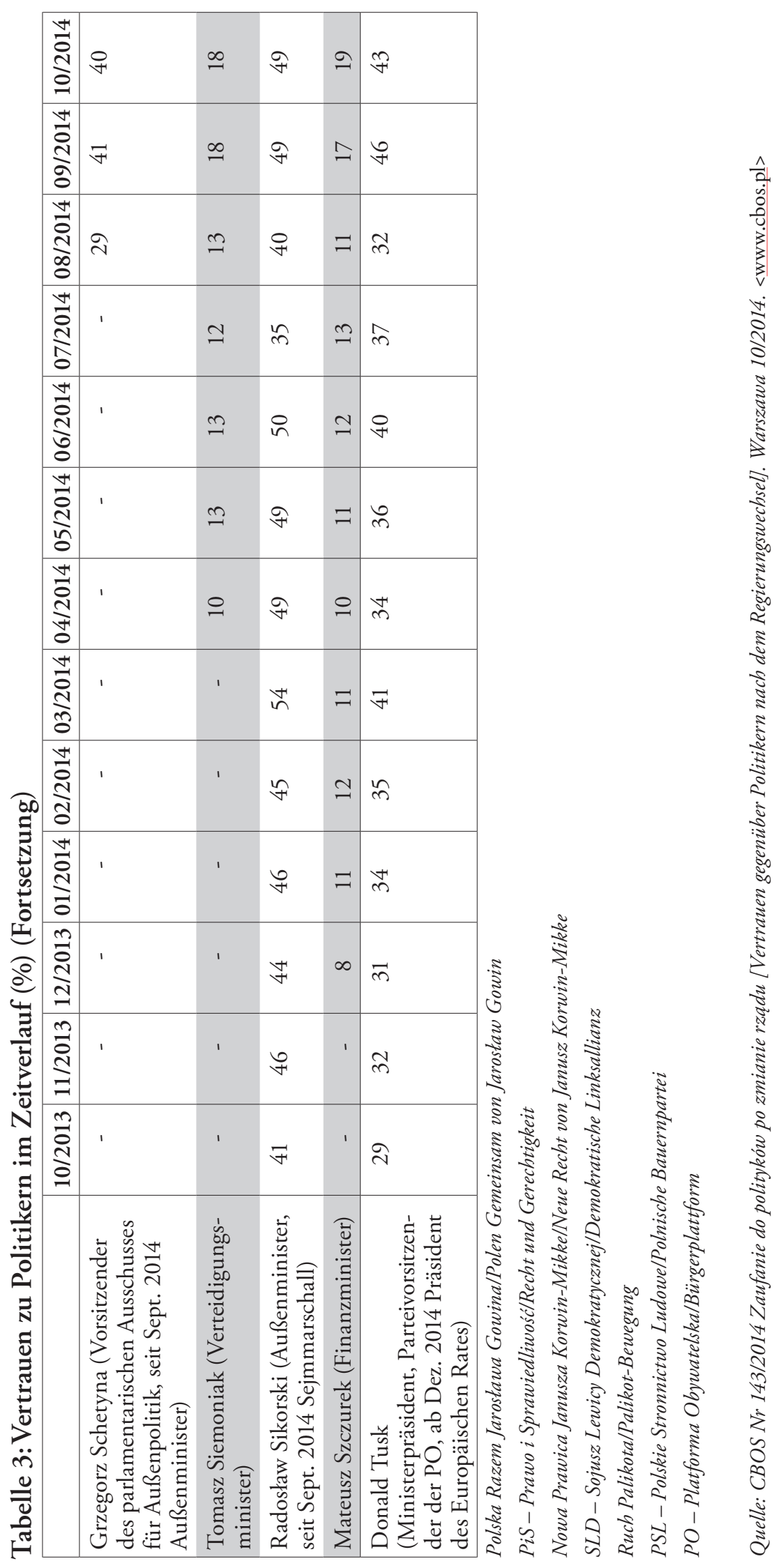


Grafik 4: Wird Ihrer Meinung nach die neue Regierung besser oder schlechter im Vergleich zur Vorgängerregierung werden?

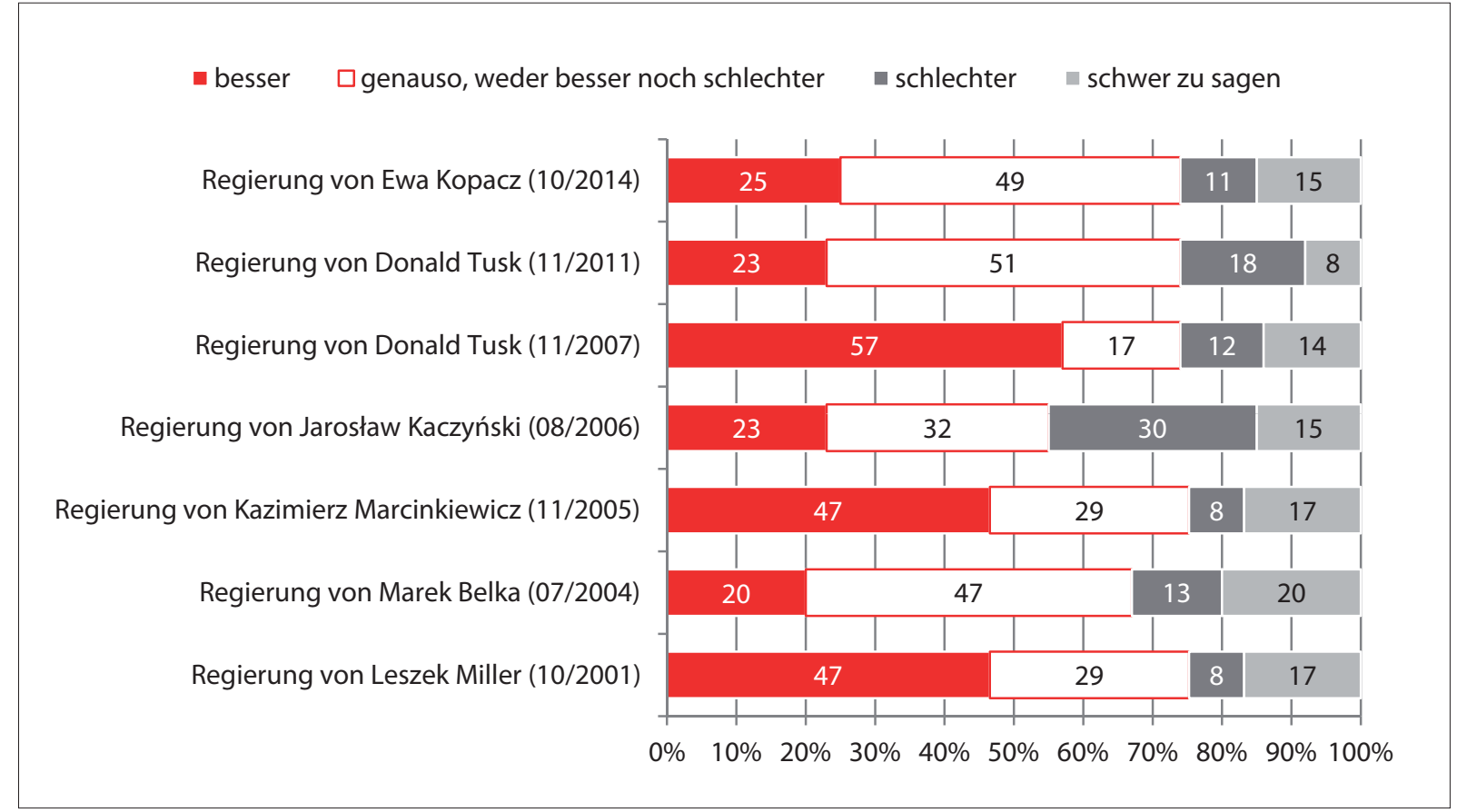

Quelle: CBOS Nr 145/2014 Pierwsze notowania rzadu Ewy Kopacz [Erste Umfragen zur Regierung von Ewa Kopacz]. Warszawa 10/2014. <www.cbos.pl>

Grafik 5: Sind Sie damit zufrieden, dass an der Spitze der Regierung ... steht? (\%)

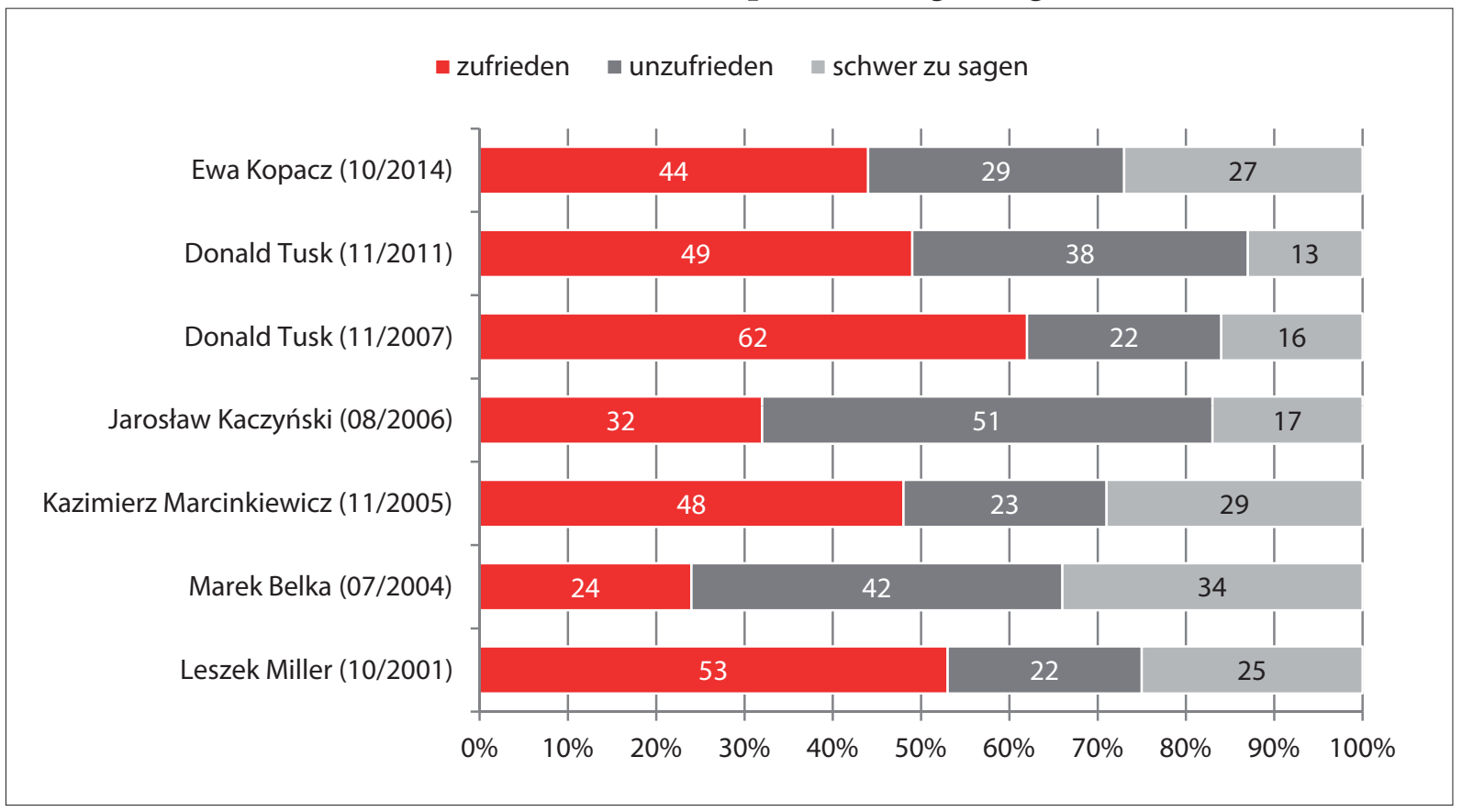

Quelle: CBOS Nr 145/2014 Pierwsze notowania rzadu Ewy Kopacz [Erste Umfragen zur Regierung von Ewa Kopacz]. Warszawa 10/2014. <www.cbos.pl> 
Grafik 6: Das Verhältnis zur Regierung* (\%)

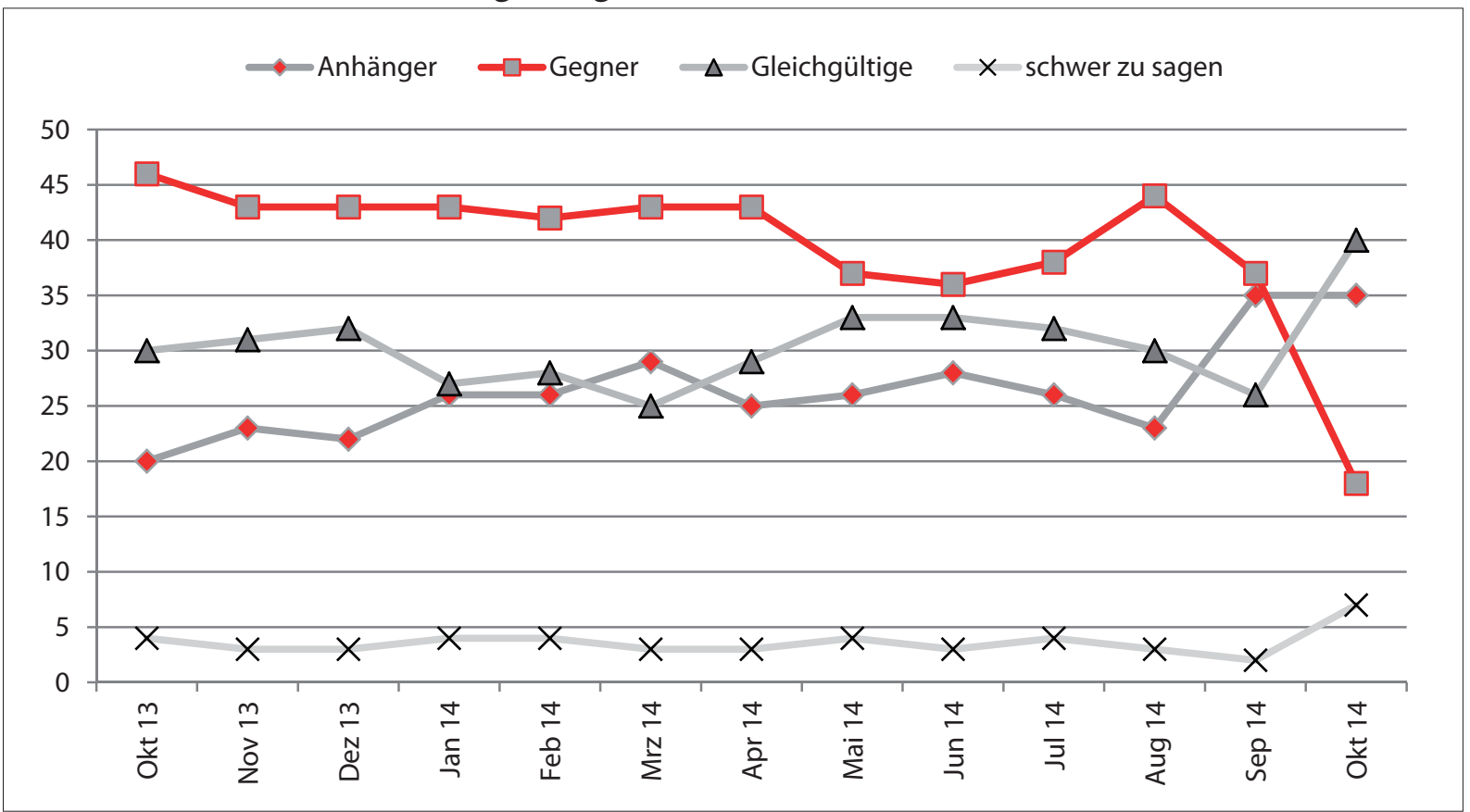

*Bis September 2014 betraf die Frage die Regierung von Donald Tusk, ab Oktober 2014 die Regierung von Ewa Kopacz.

Quelle: CBOS Nr 145/2014 Pierwsze notowania rządu Ewy Kopacz [Erste Umfragen zur Regierung von Ewa Kopacz]. Warszawa 10/2014. <www.cbos.pl> 


\section{Oktober - 3. November 2014}

\begin{tabular}{|c|c|}
\hline 21.10 .2014 & $\begin{array}{l}\text { In den Jahren } 2010 \text { bis } 2014 \text { wurden in Polen ca. 2,5 Mrd. Zloty in die Suche nach Erdöl und Schiefergas inves- } \\
\text { tiert (davon } 2014 \text { ca. } 550 \text { Mio. Zloty für Tiefenbohrungen und Frakturierungen), so der Vorsitzende der Organi- } \\
\text { sation der Polnischen Explorations- und Förderindustrie (Organizacja Polskiego Przemysłu Poszukiwawczo-Wydo- } \\
\text { bywczego), Kamlesh Parmar, auf einer für die Branche veranstalteten Konferenz. Die geologischen Erfordernisse } \\
\text { und die unklare Situation der rechtlichen Regulierungen hätten dazu geführt, dass sich die Suche verlangsamt habe. }\end{array}$ \\
\hline 22.10 .2014 & $\begin{array}{l}\text { Nach der Sitzung des Nationalen Sicherheitsrats (Rada Bezpieczeństwa Narodowego - RBN) beurteilt es der Sekre- } \\
\text { tär des RBN und Chef des Büros für Nationale Sicherheit (Biuro Bezpieczeństwa Narodowego - BBN), Stanisław } \\
\text { Koziej, als wichtigste Strategie, Reaktionsszenarien auch für Vorfälle zu entwickeln, die nicht als herkömmli- } \\
\text { cher Krieg zu bezeichnen sind. Der russisch-ukrainische Konflikt habe gezeigt, wie weitreichend auch verdeckte } \\
\text { Angriffsmethoden sein können. Eine Verteidigungsdirektive sollte neben den Verteidigungsstrukturen auch alle } \\
\text { nichtmilitärischen Ministerien und auch Woiwoden umfassen. }\end{array}$ \\
\hline 23.10 .2014 & $\begin{array}{l}\text { Auf einer Tagung für Unternehmer und Vertreter der Selbstverwaltungsorgane in Krakau plädiert die am Vortag } \\
\text { bestätigte EU-Kommissarin für Binnenmarkt, Industrie und Unternehmertum, Elżbieta Bieńkowska, dafür, den } \\
\text { Beitritt Polens zur Eurozone voranzutreiben. Die Länder der Eurozone würden stärker zusammenrücken und es sei } \\
\text { für Polen wichtig, dass es sich nicht außerhalb des internen Informationsumlaufs befinde. Es bestehe die Gefahr, } \\
\text { dass Programme umgesetzt würden, die nur die Eurozone beträfen. }\end{array}$ \\
\hline 24.10 .2014 & $\begin{array}{l}\text { Nach dem Treffen des EU-Rates (Regierungs- und Staatschefs der EU-Mitgliedsländer) in Brüssel zeigt sich Minis- } \\
\text { terpräsidentin Ewa Kopacz zufrieden über die Verhandlungsergebnisse in Klimafragen. Polen habe seine angestreb- } \\
\text { ten Ziele erreicht. Es werde keine zusätzlichen Belastungen für Polen im Zusammenhang mit den langfristigen } \\
\text { Klimaschutzzielen geben. Die Reduktion des Kohlendioxidausstoßes auf } 40 \% \text { bis zum Jahr } 2030 \text { (im Vergleich zu } \\
\text { 1990) soll aufrechterhalten werden und die Vorgaben für Energieeinsparungen sollen bei } 27 \% \text { (statt } 30 \% \text { ) liegen. }\end{array}$ \\
\hline 26.10 .2014 & $\begin{array}{l}\text { Auf einer Wahlkampfveranstaltung von Recht und Gerechtigkeit (Prawo i Sprawiedliwość - PiS) vor den Selbst- } \\
\text { verwaltungswahlen am 16. November wirft Parteichef Jarosław Kazcyński der Polnischen Bauernpartei (Polskie } \\
\text { Stronnictwo Ludowe - PSL) vor, eine Politik zu betreiben, die gegen die polnische Landwirtschaft gerichtet sei. } \\
\text { Nur PiS habe ein geeignetes Konzept zur Entwicklung des ländlichen Raums und zur Angleichung des Lebensni- } \\
\text { veaus auf dem Land und in der Stadt. }\end{array}$ \\
\hline 27.10 .2014 & $\begin{array}{l}\text { Verteidigungsminister Tomasz Siemoniak gibt bekannt, dass Polen tausende Soldaten sowie militärisches Gerät } \\
\text { von der polnischen West- an die Ostgrenze verlegen will. Hintergrund ist der russisch-ukrainische Konflikt in } \\
\text { der Ostukraine. }\end{array}$ \\
\hline 28.10 .2014 & $\begin{array}{l}\text { Der Staatspräsident Israels, Reuven Rivlin, beginnt seinen zweitägigen offiziellen Besuch in Polen. Mit seinem } \\
\text { Amtskollegen Bronisław Komorowski werden Fragen der Zusammenarbeit in der Verteidigungspolitik, bei wis- } \\
\text { senschaftlichen Forschungen sowie wirtschaftliche Themen besprochen. Am Nachmittag eröffnen beide Präsiden- } \\
\text { ten in Warschau das Museum der Geschichte der Juden in Polen. Für den nächsten Tag sind Treffen mit Minis- } \\
\text { terpräsidentin Ewa Kopacz und Sejmmarschall Radosław Sikorski vorgesehen. }\end{array}$ \\
\hline 29.10 .2014 & $\begin{array}{l}\text { In seiner Gastrede auf der Bundeswehrtagung } 2014 \text { in Berlin sagt Verteidigungsminister Tomasz Siemoniak, dass } \\
\text { eine gemeinsame Sicherheitspolitik politisch glaubwürdig und militärisch wirksam sein müsse. Wer demokratische } \\
\text { Werte nicht achte und Menschenrechte verletze, würde sich gegen den Westen und seine Werte stellen. Schwäche } \\
\text { und Unterwürfigkeit würden potentielle Aggressoren provozieren. Bundesverteidigungsminister Ursula von der } \\
\text { Leyen bezeichnet das Sicherheitsverständnis Polens als wesentlich für das deutsche und sichert Polen volle Unter- } \\
\text { stützung zu. Am Rande der Tagung unterzeichnen Frau von der Leyen und Siemoniak einen Vertrag über die } \\
\text { Intensivierung der Zusammenarbeit der deutschen und polnischen Landstreitkräfte. }\end{array}$ \\
\hline 30.10 .2014 & $\begin{array}{l}\text { Staatspräsident Bronisław Komorowski hält im Rahmen seines dreitägigen Staatsbesuches in Italien einen Vortrag } \\
\text { an der Verteidigungsakademie der NATO in Rom. Der NATO-Gipfel in Warschau } 2016 \text { sei geeignet, um einen } \\
\text { langfristigen strategischen Umbau der NATO in Gang zu setzen. Dieser sei notwendig, da sich die Krise an der } \\
\text { Ostflanke der NATO als dauerhaft erweise. Auch eine zeitweilige Pause im russisch-ukrainischen Konflikt könne } \\
\text { nicht darüber hinwegtäuschen, dass Russland den Konflikt wieder in Gang setzen werde, wenn es dies als vorteil- } \\
\text { haft erachte. Des Weiteren könne der "Islamische Staat« über den Nahen Osten hinaus auch zu einer Gefahr für } \\
\text { die EU und die NATO werden. }\end{array}$ \\
\hline 31.10 .2014 & $\begin{array}{l}\text { Nach neuesten Angaben von Eurostat betrug die Arbeitslosenquote in Polen im September 8,7 \% im Vergleich } \\
\text { zu 8,8 \% im August. Nach Berechnungen des Statistischen Hauptamtes (Głowny Urząd Statystyczny - GUS) lag } \\
\text { die Quote bei } 11,5 \% \text { (August 11,7\%). }\end{array}$ \\
\hline 03.11 .2014 & $\begin{array}{l}\text { Ministerpräsidentin Ewa Kopacz lädt die Parteivorsitzenden zur Diskussion über die Einführung eines »Bürger- } \\
\text { budgets« für jede Gemeinde ein. Dies könnte Anlass für eine Änderung des gemeinsamen Umgangs in Richtung } \\
\text { parteiübergreifende Zusammenarbeit sein, wie sie es in ihrem Exposé angemahnt habe, so Ewa Kopacz. }\end{array}$ \\
\hline
\end{tabular}

Sie können die gesamte Chronik seit 2007 auch auf < http://www.laender-analysen.de/polen/> unter dem Link »Chronik«lesen. 
Die Polen-Analysen erscheinen zweimal monatlich als E-Mail-Dienst. Sie werden gemeinsam vom Deutschen PolenInstitut Darmstadt, der Bremer Forschungsstelle Osteuropa und der Deutschen Gesellschaft für Osteuropakunde herausgegeben.

Ein Archiv der Polen-Analysen finden Sie im Internet unter <www.laender-analysen.de/polen> Kostenloses Abonnement unter <http://www.deutsches-polen-institut.de/Newsletter/subscribe.php $>$

Diese Analysen finden Sie online als Lizenzausgabe auf $<$ bpb.de>

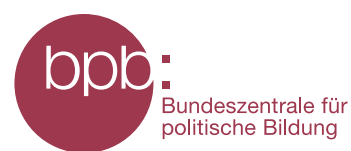

\section{Deutsches Polen-Institut Darmstadt}

Das Deutsche Polen-Institut Darmstadt (DPI) ist ein Forschungs-, Informations-, und Veranstaltungszentrum für polnische Kultur, Geschichte, Politik, Gesellschaft und die deutsch-polnischen Beziehungen, die sich im Kontext der europäischen Integration entwickeln. Das seit März 1980 aktive und bis 1997 von Gründungsdirektor Karl Dedecius geleitete Institut ist eine Gemeinschaftsgründung der Stadt Darmstadt, der Länder Hessen und Rheinland-Pfalz sowie des Bundes. 1987 wurden die Kultusminister der Länder und 2011 das Auswärtige Amt weitere institutionelle Träger. Einen wesentlichen Beitrag zur Verwirklichung der Institutsziele leisten private Stiftungen. Das DPI hat satzungsgemäß die Aufgabe, durch seine Arbeit zur Vertiefung der gegenseitigen Kenntnisse des kulturellen, geistigen und gesellschaftlichen Lebens von Polen und Deutschen beizutragen.

Ziel der Vermittlertätigkeit des DPI ist es, »die zu interessieren, auf die es politisch, wirtschaftlich, gesellschaftlich und kulturell im deutsch-polnischen Verhältnis ankommt" (Leitlinien 1997). Es geht um die Entscheider und Multiplikatoren in Politik, Kultur, Bildung, Verwaltung, Medien und Wirtschaft und, wesentlich stärker ausgeprägt als bisher, um das Hineinwirken in Wissenschaft, Forschung und Bildung.

Derzeit bemüht sich das DPI in Kooperation mit den verstreuten Orten wissenschaftlicher Polen-Kompetenz an deutschen Hochschulen und Forschungsinstituten verstärkt darum, ausgehend von einer Bestandsaufnahme deutscher Polen-Forschung Ort wissenschaftlicher Forschung und verbindendes, vernetzendes und kooperierendes Zentrum zu werden. Ausgangspunkt der Neuausrichtung ist die kaum mehr kontrollierbare Dynamik des Rückbaus der Ressourcen der wissenschaftlichen Polen-Kompetenz in den unterschiedlichen Disziplinen. Mit der über 60.000 Bände zählenden multidisziplinären Fachbibliothek für Polen, die eine einzigartige Sammlung polnischer Literatur in der Originalsprache und in deutscher Übersetzung umfasst, ist das DPI bereits ein geschätzter Ort der Recherche und des wissenschaftlichen Arbeitens. (www.deutsches-polen-institut.de)

Forschungsstelle Osteuropa an der Universität Bremen (www.forschungsstelle.uni-bremen.de)

1982 gegründet, widmet sich die Forschungsstelle Osteuropa an der Universität Bremen der interdisziplinären Analyse der Länder Ost- und Ostmitteleuropas in Zeitgeschichte und Gegenwart. Der Forschungsschwerpunkt liegt dabei auf der Rolle von "Dissens und Konsens«, von Opposition und Zivilgesellschaft in ihrem historischen, politischen, gesellschaftlichen und kulturellen Kontext. Die Forschungsstelle besitzt in ihrem Archiv eine einzigartige Sammlung alternativer Kulturgüter und unabhängiger Texte aus den ehemaligen sozialistischen Ländern. Darunter befindet sich auch eine umfangreiche Sammlung des "Zweiten Umlaufs«, die das Schrifttum und Dokumente unabhängiger Initiativen und gesellschaftlicher Gruppen in Polen aus der Zeit von 1976 bis zum Umbruch umfasst. Hinzu kommt eine umfangreiche Bibliothek mit wissenschaftlicher Literatur. Mit Archiv, Bibliothek und zwei wissenschaftlichen Abteilungen ist die Forschungsstelle auch eine Anlaufstelle sowohl für Gastwissenschaftler als auch für die interessierte Öffentlichkeit.

Eine der Hauptaufgaben der Forschungsstelle ist die Information der interessierten Öffentlichkeit. Dazu gehören unter anderem regelmäßige E-Mail-Informationsdienste für Politik, Wirtschaft, Zivilgesellschaft und Medien.

Die Meinungen, die in den Polen-Analysen geäußert werden, geben ausschließlich die Auffassung der Autoren wieder.

Abdruck und sonstige publizistische Nutzung sind nach Rücksprache mit der Redaktion gestattet.

Redaktion: Prof. Dr. Dieter Bingen (verantwortlich) (Darmstadt), Silke Plate, M.A. (Bremen) Technische Gestaltung: Matthias Neumann

Polen-Analysen-Layout: Cengiz Kibaroglu, Matthias Neumann

Alle Ausgaben der Polen-Analysen sind mit Themen- und Autorenindex archiviert unter www.laender-analysen.de

Die Polen-Analysen werden im Rahmen der Datenbank World Affairs Online (WAO) ausgewertet und sind im Portal IREON www.ireon-portal.de recherchierbar.

ISSN 1863-9712 @ 2014 by Deutsches Polen-Institut Darmstadt und Forschungsstelle Osteuropa, Bremen

Kontakt: Dr. Andrzej Kaluza, Presse- und Öffentlichkeitsarbeit, Deutsches Polen-Institut, Mathildenhöhweg 2,

D-64287 Darmstadt, Tel.: 06151/4985-13, Fax: 06151/4985-10, E-Mail: polen-analysen@dpi-da.de, Internet: www.laender-analysen.de/polen 